\title{
Recurrence Spectroscopy in Time-Dependent Fields
}

M. R. Haggerty

John B. Delos

William \& Mary, jbdelos@wm.edu

Follow this and additional works at: https://scholarworks.wm.edu/aspubs

Part of the Physics Commons

\section{Recommended Citation}

Haggerty, M. R. and Delos, John B., Recurrence Spectroscopy in Time-Dependent Fields (2000). Physical Review A, 61(5).

https://doi.org/10.1103/PhysRevA.61.053406

This Article is brought to you for free and open access by the Arts and Sciences at W\&M ScholarWorks. It has been accepted for inclusion in Arts \& Sciences Articles by an authorized administrator of W\&M ScholarWorks. For more information, please contact scholarworks@wm.edu. 


\title{
Recurrence spectroscopy in time-dependent fields
}

\author{
M. R. Haggerty \\ Department of Physics, Harvard University, Cambridge, Massachusetts 02138 \\ J. B. Delos \\ Physics Department, College of William and Mary, Williamsburg, Virginia 23187
}

(Received 22 September 1999; published 11 April 2000)

\begin{abstract}
Closed-orbit theory is a semiclassical technique for explaining the spectra of Rydberg atoms in external fields. We derive an alternative version of closed-orbit theory that applies when the external fields are time dependent. We compare the results of this theory with experiments on lithium atoms in a weak oscillating electric field.

PACS number(s): 32.60.+i, 03.65.Sq, 05.45.- a
\end{abstract}

\section{INTRODUCTION}

Recurrences are classical orbits of an electron (or quantum wave packets) that go out from an atom and later return. They are visible in real time, and they are also visible as peaks in the Fourier transform of the absorption spectrum of the atom [1-8]. In a recent experiment, the absorption spectrum of the lithium atom was measured, first with a static electric field [8], then with the static field plus a weak oscillating rf field parallel to the static field [9]. With the static field only, many strong recurrences were visible. As the rf field was added, these recurrences weakened according to a complicated pattern. One might expect that orbits having periods rationally related to the period of the driving field would be most affected, that they would be destabilized, and that those recurrences might be most weakened by the rf field. However, the experiment showed that recurrences that were "in resonance" with the driving field were least affected by the driver.

Reference [9] showed the experimental results and gave a brief explanation for the pattern of weakening caused by the rf field. Reference [10] showed that a study of the weakening allows the classical trajectories of the electrons to be reconstructed from experimental data. In this paper we present a full analysis of this experiment. Starting from the timedependent Schrödinger equation, and proceeding systematically, we derive formulas for the absorption spectrum and the recurrence spectrum in oscillating fields.

Here is a summary of the essential ideas. An atom is placed in a static field with a low-frequency oscillating field, and a laser shines on the atom. We consider the total rate of absorption of photons from the laser field, i.e., the total rate of production of electrons in highly excited states. That production rate $R_{x}\left(E_{\text {out }}, t\right)$ is an oscillatory function of time and photon energy.

The production rate oscillates with photon energy because of recurrences. As the laser acts upon the atom it produces a steady stream of outgoing electron waves with a fixed outgoing energy $E_{\text {out }}=E_{i}+h \nu$. These waves can be followed by following classical paths of the electron. The paths are turned around by the fields, and some paths return to the atom. Paths that arrive at a given final time $t$ interfere with each other and with the outgoing wave. This interference produces oscillations in the absorption rate as a function of energy $E_{\text {out }}$. The phase of the oscillations is related to the classical action around the orbit, $S$, which depends on the outgoing energy. The absorption rate $R_{x}(t)$ is proportional to $\sin (S / \hbar)$.

Now, because the applied fields are oscillating, the returning orbits likewise oscillate in time, and their classical actions also must oscillate as a function of their arrival time, $S=S\left(E_{\text {out }}, t\right)$. In first order, there is a small shift in the action, proportional to the amplitude of the oscillating field: $S\left(E_{\text {out }}, t\right)=S_{0}\left(E_{\text {out }}\right)+$ const $\times \sin (\omega t)$. Then the instantaneous absorption rate $R_{x}(t)$ depends on photon energy and time as

$$
\begin{aligned}
R_{x}\left(E_{\text {out }}, t\right) & \propto \sin \left[S\left(E_{\text {out }}, t\right) / \hbar\right] \\
= & \sin \left\{\left[S_{0}\left(E_{\text {out }}\right)+\operatorname{const} \times \sin (\omega t)\right] / \hbar\right\} .
\end{aligned}
$$

The measurement averages over many rf cycles. When we average the absorption rate over time, its oscillatory dependence on $E_{\text {out }}$ is weakened, in proportion to a Bessel function. The argument of that Bessel function turns out to be proportional to the amplitude of the oscillating field, and to the frequency-dependent dipole moment of the closed orbit. In essence, the time-dependent field causes the phase of the returning electron wave to be frequency modulated, weakening its Fourier component at the frequency corresponding to $E_{\text {out }}$. This phenomenon explains the measurements.

Our derivation follows in spirit the analysis of recurrences in static fields developed earlier by Du and Delos [1]. However, they assumed that the fields imposed on the atom were static, and they developed their whole theory from the fixedenergy Green's function. Therefore, to examine absorption spectra and recurrence spectra in oscillating fields, we have to start over from the beginning, and develop the whole theory in a time-dependent framework. The theory can be derived from a formula that happened to appear in Ref. [1] as Eq. (2.16a):

$$
\begin{aligned}
D f\left(E_{f}\right)= & {\left[\frac{2 m_{e}\left(E_{f}-E_{i}\right)}{\pi \hbar^{3}}\right] \operatorname{Re} \int_{0}^{\infty}\left\langle D \psi_{i}\left|\hat{K}^{+}(t, 0)\right| D \psi_{i}\right\rangle } \\
& \times \exp \left(i E_{f} t / \hbar\right) d t .
\end{aligned}
$$


In that paper, this formula was mentioned as an "aside" -it was not really essential to the theory, and the proof of this equation given in Ref. [1] presumed that the applied fields were time independent. Here we show that a similar formula also applies to our time-dependent system. The assumptions used in our derivation are quite general; the primary assumption is that the atom is acted upon by two fields that are far apart in frequency.

The theory developed in this paper has connections with other work on a number of other topics in atomic and molecular physics. (1) Real-time recurrences [7]: Recurrences have also been observed in real time. A short pulse of light applied to an atom creates an electron wave packet; this packet moves away from the atom, and it is detectable when it returns to the atom. However, all such measurements to date have involved only static fields applied to the atom. (2) Two-color excitation [11]: There are many observations and calculations dealing with the excitation of atoms by two coherent sources having different frequencies. The experiments discussed here are of the same type. The special features in the present case are that (i) one field is very slow (rf) and the other is very fast (visible); and (ii) the excitation is to highRydberg states, corresponding to principal quantum number around 110. (3) Microwave ionization [12]: In the experiments of Bayfield, Koch, and their groups, hydrogen atoms were excited to states around $n \sim 30-60$, and then the excited atoms were passed through a microwave cavity. In the experiments of Gallagher, sodium atoms in the ground state entered a microwave cavity, and there the laser excited them to high states. (The different order of events is significant.) The experiments considered here [9] are similar to this second case - the laser excitation occurs within the oscillating rf field. However, these experiments have a number of new aspects: (i) high resolution is achieved; (ii) the laser is coherent on a time scale which is long compared to a cycle of the rf field, and, more important, long compared to the return times of many orbits of the electron; and (iii) the experiments are done by the scaled-variable method (which is optimal for recurrence spectroscopy). Therefore recurrences can be observed, and their actions and strengths can be measured quantitatively.

\section{A. Physical picture}

To avoid inessential complications, we develop a theory for a "pseudohydrogen" atom: spin is ignored; the atom has just one active electron; in the excited states we ignore quantum defects and their associated phase shifts; and the initial state is a product of a radial factor times a spherical harmonic. The method for describing real lithium (with its phase shifts and core scattering) has been given elsewhere [3], and we do not need to be distracted by those details. In the experiment, static, rf, and laser fields are all oriented along the $z$ axis; therefore, the system is cylindrically symmetric. Moreover, $m=0$ throughout [13]. Therefore the azimuthal angle $\phi$ is ignorable, and the spatial variables are $\mathbf{q}$ $=(r, \theta)$.

The Hamiltonian has a number of terms, which we present in their approximate order of importance. The atomic Hamiltonian is

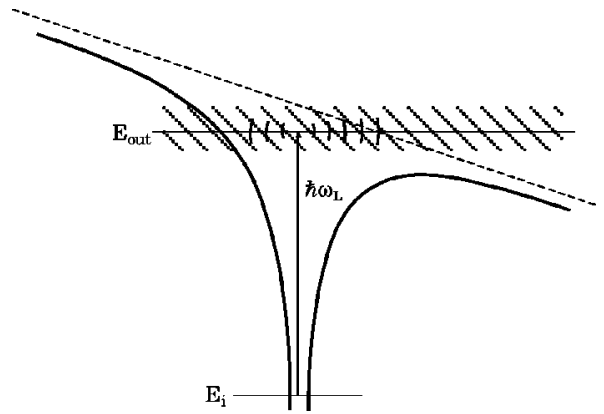

FIG. 1. Atoms are excited from an initial state of a sharply defined energy $E_{i}$ by a steady, coherent laser of a sharply defined frequency $\omega_{L}$, and electron waves of energy $E_{\text {out }}=E_{i}+\hbar \omega_{L}$ propagate outward. A rf field acts upon the outgoing electrons, changing their energy within some range. The static and rf fields have little effect on the initial state.

$$
H_{\text {atomic }}=\frac{\mathbf{p}^{2}}{2 m_{e}}-\frac{e^{2}}{r}+V_{\text {core }}(r),
$$

but we shall neglect the term $V_{\text {core }}$ due to the ionic core. Applied to the atom is a static electric field on the $z$ axis,

$$
H_{\text {static }}=H_{\text {atomic }}+e F_{0} z,
$$

and a weaker rf field: also polarized parallel to the $z$ axis;

$$
\begin{aligned}
& H(t)=H_{\text {static }}+H_{\mathrm{rf}}(t), \\
& H_{\mathrm{rf}}(t)=e F_{1} z \sin (\omega t) .
\end{aligned}
$$

Finally there is a laser field, also polarized parallel to $z$, having a high frequency $\omega_{L}$ :

$$
\begin{aligned}
H_{L}(t) & =2 e F_{L} \cos \left(\omega_{L} t+\gamma_{L}\right) D_{z} \\
& \rightarrow e F_{L} e^{-i\left(\omega_{L} t+\gamma_{L}\right)} D_{z} .
\end{aligned}
$$

$D_{z}$ is the dipole operator describing the laser's polarization. In the experiment, the laser is linearly polarized along the $z$ axis, so $D_{z}=z$. As usual, since we are only interested in excitation, we keep only the "positive-energy" term of the cosine. The phase of the laser relative to that of the rf field is $\gamma_{L}$, which will be shown later to be unimportant. Our assumptions are as follows (Fig. 1).

(1) Initially, atoms are prepared in a low-energy eigenstate of $H_{\text {atomic }}$,

$$
H_{\text {atomic }}\left|\psi_{i}\right\rangle=E_{i}\left|\psi_{i}\right\rangle
$$

At laboratory field strengths, these compact states are negligibly affected by the external fields. In our particular case, the electron has been prepared in the $3 s$ state of lithium, so $\psi_{i}(\mathbf{q})$ is the radial $3 s$ wave function $R_{3 s \mathrm{Li}}(r) /(4 \pi)^{1 / 2}$. (For true hydrogen atoms, the static field would break the degeneracy of the atomic states, and the appropriate initial states would be eigenfunctions of $H_{\text {static }}$ such as $2 s \pm 2 p$.) (2) We assume that the low-frequency field $H_{\mathrm{rf}}(t)$ has a negligible effect on the initial state. In particular, we assume that it is not strong enough to cause transitions directly into or out of 
the initial state. (3a) The laser has a well-defined frequency $\omega_{L}$ which is of course much larger than the rf frequency $\omega$ (in our case $10^{14}$ compared to $10^{8} \mathrm{~Hz}$ ). Furthermore, the laser frequency is sharp, $\delta \omega_{L} \ll \omega$, so the laser is coherent on a time scale that is long compared to the cycles of the rf field. This is important because this type of recurrence spectroscopy involves the observation of interference between outgoing and returning waves: therefore, the laser must be coherent on a time scale longer than the return time of classical orbits. (3b) We further assume that $H_{L}(t)$ is "weak," and can be treated in first-order time-dependent perturbation theory. A consequence will be that it steadily pumps electron probability from the initial state to the high-energy states, and that de-excitation is neglected.

To formulate equations with good time limits, we idealize the experiment to the following sequence of events. (i) The atoms begin "at rest," with the electron prepared in a lowenergy initial state $\psi_{i}(\mathbf{q})$. (ii) The rf field is turned on, but has negligible effect. (iii) The laser field is turned on, and left on for many cycles of the rf field; it excites some atoms to high-energy states. (iv) A pulsed electric field is applied that collects all electrons in all high-energy states, and these electrons (or the residual ions) are counted. (The pulsed field does not ionize atoms that remain in the initial state.) The measurement records the number of electrons collected (i.e., the probability that an electron has made a transition into any high-energy state) divided by the time that the laser acts upon the electron-that is, one measures the average rate of production of high-energy electrons by the laser field.

\section{B. Excitation rate}

At any time $t$, there is some probability that the electron has survived in the initial state, and some probability that it has been excited. Let us write the full wave function of the system as the sum of two terms, one representing the initial state and the other representing "all the rest":

$$
|\Psi(t)\rangle=e^{-i E_{i} t / \hbar}\left|\psi_{i}\right\rangle+\left|\Psi_{x}(t)\right\rangle .
$$

The label " $x$ " means "excited"; this term represents the development of electron probability in the entire band of highly excited states.

The experiment subjects the atom to the laser interaction for a fixed length of time $T_{\text {int }}$ (long compared with the rf period), then measures the total probability $\left\langle\Psi_{x}\left(T_{\text {int }}\right) \mid \Psi_{x}\left(T_{\text {int }}\right)\right\rangle$ that the atom has been excited. The experimentally measured average rate of excitation is then

$$
\bar{R}_{x} \equiv \frac{1}{T_{\mathrm{int}}}\left\langle\Psi_{x}\left(T_{\mathrm{int}}\right) \mid \Psi_{x}\left(T_{\mathrm{int}}\right)\right\rangle .
$$

We will proceed by calculating the instantaneous excitation rate,

$$
R_{x}(t) \equiv \frac{d}{d t}\left\langle\Psi_{x}(t) \mid \Psi_{x}(t)\right\rangle,
$$

and will show that $R_{x}(t)$ oscillates at the frequency of the rf field. Then we will average $R_{x}(t)$ over a rf cycle to obtain the theoretically predicted average excitation rate $\bar{R}_{x}$ :

$$
\bar{R}_{x}=\frac{1}{T_{\mathrm{rf}}} \int_{0}^{T_{\mathrm{rf}}} R_{x}(t) d t .
$$

\section{SMALL DISTANCES: QUANTUM REGIME}

The physics near the atom is dominated by the Coulomb attraction and the effect of the laser; the external fields are negligible. We treat this regime quantum mechanically.

\section{A. Effect of the laser}

The laser field is a weak perturbation which can be treated using standard methods of perturbation theory. The wave function for the electron satisfies the time-dependent Schrödinger equation

$$
i \hbar \frac{\partial}{\partial t}|\Psi(t)\rangle=\left[H(t)+H_{L}(t)\right]|\Psi(t)\rangle .
$$

To first order in $H_{L}$, and using the fact that $H(t)\left|\psi_{i}\right\rangle$ $\approx E_{i}\left|\psi_{i}\right\rangle$ (i.e., the applied fields have a negligible effect on the initial state), the excited-state wave function $\left|\Psi_{x}(t)\right\rangle$ obeys an inhomogeneous Schrödinger equation,

$$
\begin{aligned}
& {[i \hbar(\partial / \partial t)-H(t)]\left|\Psi_{x}(t)\right\rangle} \\
& \quad=\left(e F_{L}\right) e^{-i E_{i} t / \hbar} e^{-i\left(\omega_{L} t+\gamma_{L}\right)} D_{z}\left|\psi_{i}\right\rangle .
\end{aligned}
$$

It can be seen from Eq. (2.2) that $\gamma_{L}$ only contributes a constant phase to $\left|\Psi_{x}\right\rangle$, and can be set to zero. We are left with

$$
[i \hbar(\partial / \partial t)-H(t)]\left|\Psi_{x}(t)\right\rangle=|I(t)\rangle,
$$

where

$$
|I(t)\rangle \equiv\left(e F_{L}\right) e^{-i E_{\text {out }} t / \hbar} D_{z}\left|\psi_{i}\right\rangle
$$

is a source function that oscillates with the energy

$$
E_{\text {out }} \equiv E_{i}+\hbar \omega_{L},
$$

but is otherwise time independent. The source function is localized. Thus the time-dependent, spatially extended excited wave function $\left|\Psi_{x}(t)\right\rangle$ is generated by the steady, compact source $|I(t)\rangle$. As a consequence of this first-order treatment, the source function on the right-hand side of Eq. (2.3) contains the electric field from the laser, while the Hamiltonian $H(t)$ on the left-hand side contains the static and rf fields, but not the laser field.

We can express the solution to this equation as an integral containing the time propagator under $H(t)$. In operator form, this propagator $K^{+}\left(t, t^{\prime}\right)$ is the solution to the Schrödinger equation containing $H(t)$ :

$$
\begin{gathered}
{[i \hbar(\partial / \partial t)-H(t)] K^{+}\left(t, t^{\prime}\right)=0: \quad t>t^{\prime}} \\
K^{+}\left(t, t^{\prime}\right)=1: \quad t=t^{\prime}
\end{gathered}
$$




$$
K^{+}\left(t, t^{\prime}\right)=0: \quad t<t^{\prime}
$$

Then one can easily show that

$$
\left|\Psi_{x}(t)\right\rangle=(i \hbar)^{-1} \int_{-\infty}^{t} d t^{\prime} K^{+}\left(t, t^{\prime}\right)\left|I\left(t^{\prime}\right)\right\rangle,
$$

where $|I(t)\rangle$ is the source function defined in Eq. (2.4).

The starting point of the theory of recurrences in timedependent fields is the theorem

$$
R_{x}(t)=-\frac{2}{\hbar} \operatorname{Im}\left\langle I(t) \mid \Psi_{x}(t)\right\rangle .
$$

The proof is in Appendix A 2.

\section{B. Source function is localized}

To obtain a physical interpretation of the above formulas, let us write Eqs. (2.6), (2.7), and (2.4) in configuration representation:

$$
\begin{gathered}
\Psi_{x}(\mathbf{q}, t)=(-i \hbar)^{-1} \int_{-\infty}^{t} d t^{\prime} \int d \mathbf{q}^{\prime} K^{+}\left(\mathbf{q}, t ; \mathbf{q}^{\prime}, t^{\prime}\right) I\left(\mathbf{q}^{\prime}, t^{\prime}\right) \\
R_{x}(t)=-(2 / \hbar) \operatorname{Im}\left\{\int d \mathbf{q} I^{*}(\mathbf{q}, t) \Psi_{x}(\mathbf{q}, t)\right\} \\
I(\mathbf{q}, t)=\left(e F_{L}\right) \exp \left(-i E_{\text {out }} t / \hbar\right) D_{z} \psi_{i}(\mathbf{q})
\end{gathered}
$$

Equation (2.8a) tells us that the function $I(\mathbf{q}, t)$ acts as a "source function" for the waves $\Psi_{x}(\mathbf{q}, t)$ : the wave $\Psi_{x}$ at location $\mathbf{q}$ at time $t$ is the superposition of all the waves that propagated from all locations $\mathbf{q}^{\prime}$ in the source from all earlier times $t^{\prime}$. The excitation rate $R_{x}(t)$ is related to the overlap of these waves with the source function at time $t$. The source function $I(\mathbf{q}, t)$ is well localized: it is negligible everywhere except in a ball of perhaps $10 a_{0}$ around the nucleus. It follows that we primarily need to know about the behavior of $\Psi_{x}(\mathbf{q}, t)$ in the same small ball.

In this region, $\Psi_{x}(\mathbf{q}, t)$ consists of two types of waves. There are waves that propagate from $\mathbf{q}^{\prime}$ to $\mathbf{q}$ directly, without leaving the vicinity of the nucleus. The propagation time $t-t^{\prime}$ is typically less than ten atomic time units. In addition, there are waves that propagate outward from $\mathbf{q}^{\prime}$ and wander around under the influence of the long-range Coulomb field and the applied electric fields, visiting distant locations before returning to the vicinity of the nucleus and arriving at $\mathbf{q}$ $10^{5}$ or more atomic time units later. The distinction between these two types of waves is unambiguous because of the large distinction in distances and times. We name these two waves in the vicinity of the nucleus $\Psi_{x}^{\mathrm{dir}}(\mathbf{q}, t)$ and $\Psi_{x}^{\mathrm{ret}}(\mathbf{q}, t)$.

\section{Direct wave}

The direct wave $\left|\Psi_{x}^{\mathrm{dir}}\right\rangle$ is needed for two purposes. First, it enters into the expression for the smooth background part of the absorption. Second, it determines the starting ampli- tudes associated with the classical trajectories that will be used to calculate the returning wave. In this section we derive an expression for $\left|\Psi_{x}^{\text {dir }}\right\rangle$.

As stated earlier, near the atom the external fields are much weaker than the atomic Coulomb field; they have a negligible effect on the waves that go directly from $\mathbf{q}^{\prime}$ to $\mathbf{q}$ without leaving the vicinity of the atom. Therefore, to calculate the direct wave, we can approximate $K^{+}\left(t, t^{\prime}\right)$ by the atomic propagator

$$
K_{\text {atomic }}^{+}\left(t, t^{\prime}\right) \equiv \exp \left[-i\left(t-t^{\prime}\right) H_{\text {atomic }} / \hbar\right] .
$$

We substitute Eq. (2.9) into Eq. (2.6), and perform the time integration (inserting the conventional exponential cutoff factor to ensure convergence):

$$
\begin{aligned}
\left|\Psi_{x}^{\operatorname{dir}}(t)\right\rangle= & \lim _{\alpha \rightarrow 0^{+}}(i \hbar)^{-1} \int_{-\infty}^{t} d t^{\prime} \exp \left[-i\left(t-t^{\prime}\right) H_{\text {atomic }} / \hbar\right] \\
& \times e^{-\alpha\left(t-t^{\prime}\right) / \hbar}\left(e F_{L}\right) \exp \left[-i E_{\text {out }} t^{\prime} / \hbar\right] D_{z}\left|\psi_{i}\right\rangle \\
= & \left(e F_{L}\right) e^{-i E_{\text {out }} t / \hbar}\left[E_{\text {out }}-H_{\text {atomic }}+i \alpha\right]^{-1} D_{z}\left|\psi_{i}\right\rangle \\
= & \left(e F_{L}\right) e^{-i E_{\text {out }} t / \hbar}\left|\psi_{\text {out }}\right\rangle
\end{aligned}
$$

where

$$
\left|\psi_{\text {out }}\right\rangle \equiv G_{\text {atomic }}^{+}\left(E_{\text {out }}\right) D_{z}\left|\psi_{i}\right\rangle
$$

is a stationary wave function that appeared in earlier papers [Ref. [3], Eq. (5.8)].

We see that, except for the phase factor, $\left|\Psi_{x}^{\operatorname{dir}}(t)\right\rangle$ is a perfectly steady time-independent outgoing wave. Since the electron is acted upon by oscillating rf fields, its energy is not conserved. However, on the small scale of $\left|\psi_{i}\right\rangle$, the energy is negligibly affected by the rf field, and so it has the sharply defined value $E_{\text {out }}=E_{i}+\hbar \omega_{L}$. Thus the effect of the laser on the atom is to give locally a steady coherent stream of outgoing waves of well-defined energy $E_{\text {out }}$.

At intermediate $r$ [in the general vicinity of the atom but outside the range where $\psi_{i}(\mathbf{q})$ is significant], $\psi_{\text {out }}$ separates approximately into a product of radial and angular parts:

$$
\psi_{\text {out }}(\mathbf{q}) \approx C_{1}\left\{\frac{\exp (i \sqrt{8 r})}{r^{3 / 4}}\right\} \mathcal{Y}(\theta, \phi)
$$

The radial part is a zero-energy spherical outgoing Coulomb wave, while $\mathcal{Y}(\theta, \phi)$ describes the intensity and angular distribution of outgoing waves. $\mathcal{Y}(\theta, \phi)$ can be obtained by projecting $\left|D_{z} \psi_{i}\right\rangle$ onto a Coulomb scattering wave,

$$
\mathcal{Y}(\theta, \phi) \equiv C_{2}^{-1}\left\langle\psi_{\hat{\mathbf{k}}} \mid D_{z} \psi_{i}\right\rangle
$$

where $\left|\psi_{\hat{\mathbf{k}}}\right\rangle$ is the zero-energy Coulomb scattering wave leaving the nucleus in direction $\hat{\mathbf{k}}$,

$$
\psi_{\hat{\mathbf{k}}}(\mathbf{q}) \equiv J_{0}\left(\sqrt{8 r} \sqrt{\frac{1}{2}(1+\hat{\mathbf{k}} \cdot \hat{\mathbf{r}})}\right)
$$

and $\hat{\mathbf{k}}$ points in direction $(\theta, \phi)$. Since the initial state is cylindrically symmetric and the laser is linearly polarized 
along the static field, $\mathcal{Y}$ does not depend on $\phi$, and we can write $\mathcal{Y}(\theta)$. For more details including the value of the constant $C_{2}$, see Appendix A 3 .

The direct contribution to the excitation rate follows from Eqs. (2.7) and (2.11):

$$
R_{x}^{\mathrm{dir}}=-\frac{2}{\hbar}\left(e F_{L}\right)^{2} \operatorname{Im}\left\langle\psi_{i}\left|D_{z}^{\dagger} G_{\text {atomic }}^{+}\left(E_{\text {out }}\right) D_{z}\right| \psi_{i}\right\rangle .
$$

One can evaluate Eq. (2.15) directly, but a shortcut is simply to calculate the flux of electrons going out through a sphere at radius $r$ large enough for Eq. (2.12) to be valid:

$$
\begin{aligned}
R_{x}^{\mathrm{dir}} & =r^{2} \int \sin \theta d \theta \int d \phi \operatorname{Im}\left\{\left[\Psi_{x}^{\mathrm{dir}}(\mathbf{q})\right]^{*} \frac{\partial}{\partial r} \Psi_{x}^{\mathrm{dir}}(\mathbf{q})\right\} \\
& =4 \pi\left(e F_{L}\right)^{2} \int \sin \theta d \theta \int d \phi|\mathcal{Y}(\theta, \phi)|^{2}
\end{aligned}
$$

Note that $R_{x}^{\mathrm{dir}}$ is time independent, and it is the same smooth, nearly energy-independent background that was obtained in Ref. [1].

\section{LARGE DISTANCES: SEMICLASSICAL REGIME}

For large distances, the effect of the laser is negligible and the physics is dominated by the external electric fields and the Coulomb field. We treat this regime semiclassically.

\section{A. Continuing the wave function semiclassically}

In Sec. II C, we showed how to find an approximate wave function describing the outgoing electron wave near the nucleus. Our task now is to propagate this wave function through large distances and back to the nucleus. We do this semiclassically, by following classical trajectories from the region near the nucleus, where the outgoing wave function is known, to the regions where we want to compute the returning wave function. We will follow the method of Maslov and Fedoriuk [14], as done in Ref. [1], but with the difference that our system is time dependent. Proofs of the formulas used in this section can be found in Appendix B.

The recipe requires that we know the outgoing wave function on some surface in configuration space, for all time. This condition is met if we take this initial surface to be a sphere of radius $r_{i} \sim 10 a_{0}$, where $\Psi_{x}^{\mathrm{dir}}$ is known from Eq. (2.10). To find the wave function in the rest of space, we imagine that classical trajectories depart radially outwards from this sphere, starting at all initial angles and all initial times, and with initial energy $E_{\text {out }}$. The trajectories propagate under the influence of the time-dependent Hamiltonian $H(t)$, consisting of the Coulombic attraction to the nucleus and the static and rf external fields. Where the classical electron trajectories go, so go the quantum electron waves.

A trajectory is specified by its initial angles and its initial time. The angles $\theta_{i}$ and $\phi_{i}$ indicate where on the initial sphere the trajectory began, as well as the direction of the initial momentum (we will omit $\phi$ because of the cylindrical symmetry of our system). The initial time $t_{i}$ represents the "laboratory time" when the electron emerged from the surface (e.g., 3:42 A.M.). There are two ways to specify a later point along a given trajectory: by the final laboratory time $t$ or by the "travel time" $\tau \equiv t-t_{i}$. Thus $\mathbf{q}\left(\theta_{i}, t_{i}, t\right)$ is the point that is reached at laboratory time $t$ by an electron started at time $t_{i}$ from angle $\theta_{i}$. Alternatively, $\mathbf{q}\left(\theta_{i}, t_{i} ; \tau\right)$ is the point reached by the same trajectory after it has been traveling for duration $\tau$ (e.g., $150 \mathrm{ps}$ ).

The family of classical trajectories carries the wave. If a trajectory travels from a point $\mathbf{q}_{i}$ on the initial surface at time $t_{i}$ to a point $\mathbf{q}$ at some later time $t$, then the semiclassical approximation to the excited-state wave function at that point is (see Appendix B)

$$
\begin{aligned}
\Psi_{x}^{\mathrm{sc}}(\mathbf{q}, t)= & \Psi_{x}^{\mathrm{dir}}\left(\mathbf{q}_{i}, t_{i}\right) A\left(\mathbf{q}, t ; \mathbf{q}_{i}, t_{i}\right) \\
& \times \exp \left\{i\left[\mathcal{S}\left(\mathbf{q}, t ; \mathbf{q}_{i}, t_{i}\right) / \hbar-\lambda \pi / 2\right]\right\} .
\end{aligned}
$$

[If several trajectories pass through $(\mathbf{q}, t)$, the wave function is the superposition of the contributions (3.1) from each trajectory.] This wave function is proportional to the direct outgoing wave $\Psi_{x}^{\mathrm{dir}}\left(\mathbf{q}_{i}, t_{i}\right)$ at the point on the initial sphere from which the trajectory that arrives at $(\mathbf{q}, t)$ emanated. The phase is given by Hamilton's principal function $\mathcal{S}$, integrated from $\left(\mathbf{q}_{i}, t_{i}\right)$ to $(\mathbf{q}, t)$ :

$$
\mathcal{S}\left(\mathbf{q}, t ; \mathbf{q}_{i}, t_{i}\right)=\int_{\left(\mathbf{q}_{i}, t_{i}\right)}^{(\mathbf{q}, t)}\left[\mathbf{p} \cdot d \mathbf{q}-H d t^{\prime}\right] .
$$

Compared to a time-independent treatment, the new term is $-\int H d t^{\prime}$, and we emphasize that energy is not conserved on the trajectories. The amplitude $A\left(\mathbf{q}, t ; \mathbf{q}_{i}, t_{i}\right)$ is related to the density of classical trajectories, and will be explained below. Finally, $\lambda$ is the Maslov index. In previous papers it was called $\mu$, but we change the letter because the Maslov index for a time-varying field need not be equal to that for a fixed field. The Maslov index is the number of times (including multiplicities) that the amplitude diverges on the path from the initial surface to the field point $(\mathbf{q}, t)$.

The amplitude $A\left(\mathbf{q}, t ; \mathbf{q}_{i}, t_{i}\right)$ can be computed from a ratio of Jacobians evaluated at the initial and final points:

$$
A\left(\mathbf{q}, t ; \mathbf{q}_{i}, t_{i}\right)=\left|\frac{J\left(\mathbf{q}_{i}, t_{i}\right)}{J(\mathbf{q}, t)}\right|^{1 / 2} .
$$

Several expressions for $J(\mathbf{q}, t)$ are given in Appendix B; for example,

$$
J(\mathbf{q}, t)=-r^{2} \sin \theta \operatorname{det}\left[\frac{\partial(r, \theta)}{\partial\left(\theta_{i}, t_{i}\right)}\right]_{t} .
$$

Conceptually, $J(\mathbf{q}, t)$ can be computed by comparing three trajectories. First the trajectory that arrives at $(\mathbf{q}, t)$ is identified, and its initial values $\left(\theta_{i}, t_{i}\right)$ found. Then each of $\theta_{i}$ and $t_{i}$ are perturbed slightly, and those trajectories are integrated to the same final time $t$. The Jacobian is evaluated at the field point $(\mathbf{q}, t)$ and at the corresponding point $\left(\mathbf{q}_{i}, t_{i}\right)$ on the initial sphere to obtain $A\left(\mathbf{q}, t ; \mathbf{q}_{i}, t_{i}\right)$. 


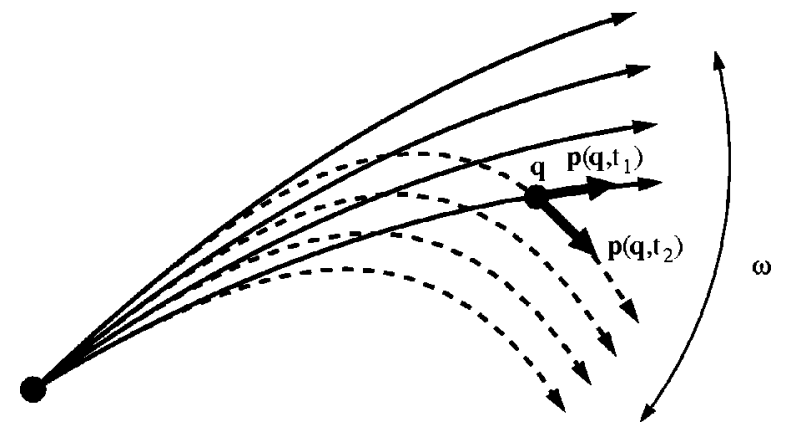

FIG. 2. The solid curves are paths of electrons that emerge from the atom at some initial time $t_{i 1}$, and the dashed lines are paths of electrons that emerge later, at $t_{i 2}$. The paths form a vector field $\mathbf{p}(\mathbf{q}, t)$. As $t_{i}$ varies, the whole vector field oscillates. Similarly, at any location $\mathbf{q}$, the vector field $\mathbf{p}(\mathbf{q}, t)$ oscillates in time $t$ with the frequency of the rf field. The vector field "supports" a semiclassical wave function $\Psi_{x}^{\mathrm{sc}}(\mathbf{q}, t)$, which is also oscillating in real time with the frequency of the $\mathrm{rf}$ field.

\section{B. Time dependence of trajectories and wave function}

The initial time $t_{i}$ has physical significance because it determines the phase of the rf field at the instant that the electron emerges from the atom. For any fixed value of the travel time $\tau, \mathbf{q}\left(\theta_{i}, t_{i} ; \tau\right)$ and $\mathbf{p}\left(\theta_{i}, t_{i} ; \tau\right)$ are periodic functions of $t_{i}$; they oscillate at the frequency of the rf field. Let us think about a pencil of trajectories that emanate from the initial sphere at time $t_{i}$ in a small range of initial directions around $\theta_{i}$ (Fig. 2).

That pencil of trajectories forms a vector field $\mathbf{p}(\mathbf{q} ; t)$. As $t_{i}$ varies and the rf field oscillates, the entire vector field wobbles in space with the same period. Close to the nucleus, the motion of the momentum-vector field is negligible (the rf field is too weak to change the initial course of the electron). But on a larger scale, the electron is traveling great distances, and the oscillating force has plenty of time to change the velocity of the electron. We might picture water coming from a fixed hose that sends out a spray in a periodically oscillating wind.

Table I summarizes the meanings of the various times that appear in the formulas. The variable $t$ is real time, as measured by a clock in the laboratory, and $\mathbf{q}$ is a point in real space. (A sufficiently small detector placed at $\mathbf{q}$ would sometimes record the presence of an actual electron.) The wave function $\Psi_{x}(\mathbf{q}, t)$ is oscillating in real time $t$ at the rf frequency. In contrast, the variables $\tau, \theta_{i}$, and $t_{i}$, and the functions $\mathbf{q}\left(\theta_{i}, t_{i} ; \tau\right)$ and $\mathbf{p}\left(\theta_{i}, t_{i} ; \tau\right)$ are "artificial" quantities that are used for the purpose of constructing a semiclassical

TABLE I. Time definitions.

\begin{tabular}{cl}
\hline \hline Symbol & Description \\
\hline$t$ & Real, laboratory time (e.g., 3:42 A.M.) \\
$T$ & Time duration of a classical closed orbit, \\
& including repetitions if any (e.g. 200ps) \\
$t_{i}(t)=t-T$ & Initial time of an orbit that ends at time t \\
$\tau$ & Progress variable for trajectories, $0 \leqslant \tau \leqslant T$ \\
\hline \hline
\end{tabular}

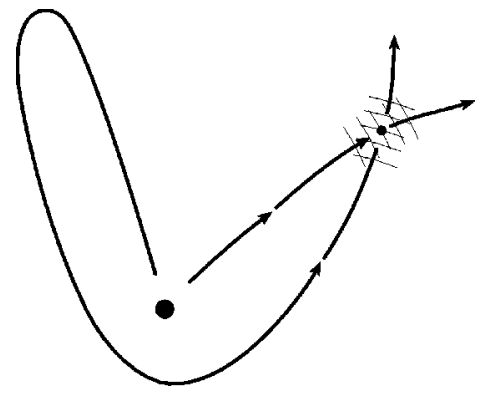

FIG. 3. If several paths arrive at a given $\mathbf{q}$ at time $t$, the wave function is a superposition of terms associated with each path. It does not matter whether the paths started at the same time-the laser has a long coherence time, so the two electron waves will nevertheless be coherent. Tracing the orbits backwards in time allows $t_{i}, \theta_{i}$, and $\tau$ for each orbit to be deduced.

approximation to the wave function $\Psi_{x}(\mathbf{q}, t)$. If an electron is seen at the location $\mathbf{q}$ at time $t$, then $t_{i}, \theta_{i}$, and $\tau$ represent the time of emergence from the initial surface, the initial direction of motion, and the travel time along the classical path that the electron would have had if it were a classical particle, respectively.

The trajectory relationship is partly invertible. At real time $t$, the "classically allowed region" is the domain of $\mathbf{q}$ space in which trajectories are present. Given some specified time $t$, and a specified point $\mathbf{q}$ in the allowed region, there is by definition at least one trajectory that goes through $\mathbf{q}$ at time $t$. We can trace that orbit backwards in time to find its initial time $t_{i}(\mathbf{q}, t)$ and initial location $\mathbf{q}_{i}(\mathbf{q}, t)$ or $\theta_{i}(\mathbf{q}, t)$ when it left the surface (see Fig. 3).

This relationship between $\left(\mathbf{q}_{i}, t_{i}\right)$ and $(\mathbf{q}, t)$ is implicit in Eq. (3.1). The travel time $\tau(\mathbf{q}, t)$ and final momentum $\mathbf{p}(\mathbf{q}, t)$ can similarly be thought of as functions of the final point $\mathbf{q}$ and time $t$. At each point $\mathbf{q}$, all of these quantities- $t_{i}(\mathbf{q}, t)$, $\theta_{i}(\mathbf{q}, t), \tau(\mathbf{q}, t)$, and $\mathbf{p}(\mathbf{q}, t)$-are periodic functions of real time $t$.

Moreover, since the entire vector field $\mathbf{p}(\mathbf{q}, t)$ is oscillating periodically in time $t$ at the $\mathrm{rf}$ frequency, it follows that at each point $\mathbf{q}$ the principal function $\mathcal{S}\left(\mathbf{q}, t ; \mathbf{q}_{i}(\mathbf{q}, t), t_{i}(\mathbf{q}, t)\right)$, the Jacobian $J(\mathbf{q}, t)$, and the classical amplitude $A\left(\mathbf{q}, t ; \mathbf{q}_{i}(\mathbf{q}, t), t_{i}(\mathbf{q}, t)\right)$ oscillate periodically as a function of time $t$ with the same frequency. But these classical quantities determine the semiclassical wave function. Thus the time dependence of the ensemble of classical orbits manifests itself as the time dependence of the wavefunction.

\section{Waves return to the atom}

According to Eq. (2.7), to find the excitation rate we need to compute the overlap of the excited wave function and the (localized) source function. Therefore, let us examine those waves that return to the general vicinity of the atom (say, within $50 a_{0}$ of the nucleus). In the semiclassical approximation, each returning wave is associated with a bundle of classical orbits that also return to the vicinity of the atom.

We now consider the generic case, with no nearby bifurcations, and for the moment we ignore the cylindrical symmetry. Under those conditions each bundle of returning tra- 
jectories contains exactly one "closed orbit" that returns exactly to the nucleus. The other trajectories in the bundle are adequately described by a linear approximation in the neighborhood of the closed orbit, which in turn can be obtained from the stability matrix of the closed orbit. We therefore can learn about the excitation rate by studying classical closed orbits.

The wave carried by a closed orbit and its neighbors may be labeled by the direction $\left(\theta_{f}, \phi_{f}\right)$ from which the closed orbit comes. For points $\mathbf{q}$ not too close to the nucleus, the semiclassical approximation (3.1) is a good representation of each such returning wave; $\Psi_{x}^{\text {ret }}(\mathbf{q}, t) \approx \Psi_{x}^{\mathrm{sc}}(\mathbf{q}, t)$. But closer to the atom, the semiclassical approximation diverges due to the Coulomb singularity, so we need to "connect" back to a quantum wave function.

Near the atom, the Coulomb field again dominates, and the quantum wave corresponding to an isolated closed orbit and its neighbors is approximately a zero-energy Coulomb scattering wave $\psi_{\hat{\mathbf{k}}_{f}}(\mathbf{q})$ [see Eq. (2.14)], returning from the same direction $\hat{\mathbf{k}}_{f}=\left(\theta_{f}, \phi_{f}\right)$ as does the classical closed orbit.

In fact, however, the system is cylindrically symmetric, so if one trajectory returns from direction $\left(\theta_{f}, \phi_{f}\right)$, then other trajectories also return from all other possible azimuths $\phi_{f}$. The appropriate quantum wave is therefore a cylindrically symmetrized zero-energy Coulomb scattering wave characterized by the angle $\theta_{f}$ :

$$
\psi_{C, \theta_{f}}(\mathbf{q}) \equiv \frac{1}{2 \pi} \int_{0}^{2 \pi} d \phi_{f} \psi_{\hat{\mathbf{k}}_{f}}(\mathbf{q})
$$

We assume that the returning wave is locally proportional to this quantum wave,

$$
\Psi_{x}^{\mathrm{ret}}(\mathbf{q}, t) \approx \Psi_{x}^{\mathrm{sc}}(\mathbf{q}, t) \approx N(t) \psi_{C, \theta_{f}}(\mathbf{q}),
$$

where $N(t)$ is some coefficient of proportionality to be determined.

Equation (3.6) expresses a functional relationship which should hold for any $\mathbf{q}$ in the region where both $\Psi_{x}^{\mathrm{sc}}$ and $\psi_{C, \theta_{f}}$ are valid, namely for intermediate distances from the atom. In Appendix A 4 we show how to determine $N(t)$ by comparing Eqs. (3.5) and (3.1):

$$
\begin{aligned}
N(t)= & e F_{L}\left(C_{1} / C_{3}\right) A_{0}(t) \mathcal{Y}\left(\theta_{i}\right) \\
& \times \exp \left\{i\left[\mathcal{S}\left(\mathbf{0}, t ; \mathbf{0}, t_{i}\right)-E_{\text {out }} t_{i}\right] / \hbar-i \lambda \pi / 2\right\} .
\end{aligned}
$$

\section{Returning waves contribute to the excitation rate}

Equation (2.7) tells us that the returning waves contribute to the excitation rate:

$$
R_{x}^{\mathrm{ret}}(t)=-\frac{2}{\hbar} \operatorname{Im}\left\langle I(t) \mid \Psi_{x}^{\mathrm{ret}}(t)\right\rangle .
$$

A straightforward sequence of substitutions gives (Appendix A 5)

$$
\begin{aligned}
R_{x}^{\mathrm{ret}}(t)= & 2^{19 / 4} \pi^{5 / 2} F_{L}^{2} A_{0}(t) \operatorname{Im}\left\{\mathcal{Y}^{*}\left(\theta_{f}\right) \mathcal{Y}\left(\theta_{i}\right)\right. \\
& \times \exp [i S(t) / \hbar-i \lambda \pi / 2-i 3 \pi / 4]\}
\end{aligned}
$$

$S(t)$ has a new definition compared to Ref. [1]: it is an "extended action," integrated all the way around the closed orbit (starting and ending at the nucleus),

$$
S(t) \equiv \int_{t_{i}(t)}^{t}\left\{\mathbf{p}\left(\mathbf{q}, t^{\prime}\right) \frac{d \mathbf{q}}{d t^{\prime}}-\left[H\left(t^{\prime}\right)-E_{\mathrm{out}}\right]\right\} d t^{\prime} .
$$

The extended action includes the usual action, $\int \mathbf{p} \cdot d \mathbf{q}$ $=\int\left(p_{r} d r+p_{\theta} d \theta\right)$, around the closed orbit, plus a new term $-\int\left[H\left(t^{\prime}\right)-E_{\text {out }}\right] d t^{\prime}$. The amplitude $A_{0}(t)$ is

$$
A_{0}(t)=\left(\sin \theta_{f} \sin \theta_{i}\right)^{1 / 2} \frac{r_{i}^{1 / 4}}{r_{f}^{1 / 2}}\left|\frac{\hat{J}\left(r_{i}, \theta_{i}, t_{i}\right)}{\hat{J}\left(r_{f}, \theta_{f}, t\right)}\right|^{1 / 2},
$$

which can be evaluated anywhere near the ends of the closed orbit. The reduced Jacobian $\hat{J}$ is defined in Eq. (B10a). The $r$ dependence of the reduced Jacobians is such that $A_{0}$ has a finite limit as $r_{i}$ and $r_{f}$ go to zero whenever approximation (3.6) for the returning wave is valid (i.e., if the closed orbit does not have a bifurcation nearby).

It is easy to show that this formula reduces to the formula that was derived for static fields [Ref. [1], Eq. (5.13a)]. If the rf field is zero, then the ratio of Jacobians is independent of time $t$, and the extra term in the extended action disappears because energy is conserved.

\section{WEAK RF FIELDS}

With the rf field acting upon the system, the whole vector field $\mathbf{p}(\mathbf{q}, t)$ oscillates periodically in time $t$ at the $\mathrm{rf}$ frequency, so the shape and size of any closed orbit oscillates with this period. The duration $T=t-t_{i}(t)$ of each closed orbit, the initial and final angles $\theta_{i}$ and $\theta_{f}$, the angular factors $\mathcal{Y}(\theta)$, the Jacobian ratio $A_{0}(t)$, and the extended action function $S(t)$ all oscillate at this frequency. It is the oscillation of $S(t)$ that produces the largest effect. In current experiments, the rf field is weak compared to the static electric field; $F_{1}$ is $1-5 \%$ of $F_{0}$. Therefore the initial and final angles $\theta_{i}$ and $\theta_{f}$ are not much affected by the rf field [and, furthermore, $\mathcal{Y}(\theta)$ is slowly varying over the whole range of $\theta$ for which orbits are closed]. Likewise, $A_{0}(t)$ and $S(t)$ oscillate by only a few percent. Since $S(t)$ is large, however, this few-percent variation of $S(t)$ is substantial compared to $\hbar$, and there can be a significant variation in the phase of the returning wave.

The theory developed up to this point applies whether the rf field is weak or strong, provided only that it is not strong enough to substantially perturb the initial state. Let us now consider a weak rf field that is a small perturbation to the dynamics in the static field, $H_{\text {static }}$. Clearly $S(t)$ is close to the value that would be obtained for static fields. How does $S(t)$ change as a result of applying the rf field? 


\section{A. Action perturbation}

Standard methods of classical mechanics (Appendix C) show that, in first order,

$$
\begin{aligned}
\Delta S\left(t, F_{1}\right) & \equiv S\left(t, F_{1}\right)-S\left(t, F_{1}=0\right) \\
& \approx-F_{1} \int_{t_{i}}^{t} \frac{\partial H}{\partial F_{1}} d t^{\prime} \\
& =-e F_{1} \int_{0}^{T} z_{0}(\tau) \sin [\omega(\tau+t-T)] d \tau,
\end{aligned}
$$

where $z_{0}(\tau)$ is the $z$ component of the electron's position on the unperturbed orbit (in the static field alone), starting (at $\tau=0$ ) and ending (at $\tau=T$ ) at the origin. Since the orbit $z_{0}(\tau)$ is calculated in the absence of the rf field, it depends only on the elapsed time $\tau$, and not on the real time $t$.

Equation (4.2) is conveniently expressed in terms of the complex, frequency-dependent ac dipole moment $Z(\omega)$ of the orbit,

$$
S(t) \approx S_{0}-e F_{1}|Z(\omega)| T \sin (\omega t+\beta)
$$

where

$$
\begin{aligned}
Z(\omega) & \equiv \frac{1}{T} \int_{0}^{T} z_{0}(\tau) \exp (i \omega \tau) d \tau, \\
\beta & =-\omega T+\arg (Z(\omega)),
\end{aligned}
$$

and $S_{0}$ is the action of the unperturbed orbit. We see that, in first order, $\Delta S$ oscillates sinusoidally in time, and it is linearly proportional to $F_{1}$, to the closure time of the orbit, and to the absolute magnitude of the ac dipole moment of the orbit.

\section{B. Averaged excitation rate}

As stated in Sec. IB, it is not the instantaneous excitation rate $R_{x}(t)$ that is observed in the experiment, but the average of $R_{x}(t)$ over a cycle. Let us calculate the contribution of the returning wave to this average by taking the time average of Eq. (3.9a). As argued in Sec. IV A, we can use for $A_{0}(t)$ its unperturbed value, which is time independent. The only significant time dependence is in $S(t)$. Using $S(t)$ given by Eq. (4.3), the integral is over one period of the sinusoid and a standard integral gives

$$
\frac{1}{T_{\mathrm{rf}}} \int_{0}^{T_{\mathrm{rf}}} d t \exp [i S(t) / \hbar]=\exp \left[i S_{0} / \hbar\right] J_{0}\left(e F_{1}|Z(\omega)| T / \hbar\right),
$$

where $J_{0}$ is a Bessel function. Note that the phase constant $\beta$ has dropped out. We can now use Eqs. (4.5), (3.9a), and (1.6) to evaluate $\bar{R}_{x}^{\text {ret }}$. The result is

$$
\begin{aligned}
\bar{R}_{x}^{\mathrm{ret}}= & 2^{19 / 4} \pi^{5 / 2} F_{L}^{2} A_{0} J_{0}\left(e F_{1}|Z(\omega)| T / \hbar\right) \operatorname{Im}\left\{\mathcal{Y}^{*}\left(\theta_{f}\right) \mathcal{Y}\left(\theta_{i}\right)\right. \\
& \left.\times \exp \left[i\left(S_{0} / \hbar-\lambda \pi / 2-3 \pi / 4\right)\right]\right\}
\end{aligned}
$$

where $\theta_{i}$ and $\theta_{f}$ are the initial and final angles of the trajectory in the static field.

If we set $F_{1}=0$ in Eq. (4.6), then $J_{0} \rightarrow 1$, and we recover the static field expression for the excitation rate. But when $F_{1}$ is turned on, the Bessel function decreases, corresponding to a weakening of the strength of the spectral oscillation caused by the closed orbit. Thus, the effect of a rf field is to weaken each recurrence from its static field value by a Bessel function factor, $J_{0}\left(e F_{1}|Z(\omega)| T / \hbar\right)$.

\section{STRONG RF FIELDS}

In Sec. IV, we introduced the assumption that the orbits are only weakly perturbed by the rf field. Let us now return to Eq. (3.9), and ask what happens as the rf field becomes stronger.

We still need to average the instantaneous excitation rate using Eq. (1.6), but now the variation of $S(t)$ with time will exceed Planck's constant, so it is appropriate to evaluate the integral by stationary phase. The stationary phase points are the extrema of $S(t)$, which satisfy

$$
\left.\frac{\partial S}{\partial t}\right|_{\mathbf{q}_{i}, \mathbf{q}_{f}}=-\left[H(t)-E_{\text {out }}\right]=0
$$

(the derivative being taken with initial and final points held fixed, $\mathbf{q}_{i}=\mathbf{q}_{f}=\mathbf{0}$ ). Therefore, the main contributions to the recurrence strength come from those closed orbits that return to the atom with the same energy they had when they left, $E_{\text {ret }}=E_{\text {out }}$. In other words, recurrences arise primarily from orbits that are closed in an extended $(\mathbf{q},-E)$ space. Such orbits form a discrete set, and can be characterized by their initial directions together with their initial times.

If we let $\hat{t}$ be the real time at return corresponding to any stationary phase point, then the resulting contribution to $\bar{R}_{x}$ is

$$
\begin{aligned}
& \bar{R}_{x}^{\mathrm{sp}}= 2^{21 / 4} \pi^{3} F_{L}^{2} \frac{A_{0}(\hat{t})}{T_{\mathrm{rf}}}\left|\frac{d E_{\mathrm{ret}}}{d t}\right|-1 / 2 \\
& \operatorname{Im}\left(\mathcal{Y}^{*}\left(\theta_{f}\right) \mathcal{Y}\left(\theta_{i}\right)\right. \\
&\left.\times \exp \left\{i S(\hat{t}) / \hbar-i \pi / 2\left[\lambda+\frac{3}{2}+\frac{1}{2} \operatorname{sgn}\left(d E_{\mathrm{ret}} / d t\right)\right]\right\}\right) .
\end{aligned}
$$

$\theta_{f}$ and $\theta_{i}$ here are the angles at the origin of the closed orbit that returns at time $\hat{t}$. $E_{\text {ret }}$ is the energy of the electron at the end of the closed orbit, when it returns to the atom; it oscillates periodically in time due to the effect of the rf field.

How does the transition from expression (4.6) (for weak rf fields) to expression (5.2) (for strong rf fields) occur? For small rf fields, we have shown that $S(t)$ is sinusoidal with a small amplitude. Equation (5.2) cannot be applied in that limit because the stationary phase approximation breaks down (because $\left|d E_{\text {ret }} / d t\right|^{-1 / 2} \rightarrow \infty$ ).

But as the sinusoid increases in amplitude, a stationary phase approximation becomes appropriate. Now $S(t)$ has two extrema - one maximum and one minimum - per period, and Eq. (5.2) can be applied to each in turn. The sum of the 
two contributions gives precisely the large-argument expansion of the Bessel function of Eq. (4.6).

As the $\mathrm{rf}$ field increases, the sinusoidal approximation breaks down, and the stationary phase expression begins to differ from Eq. (4.6). For still stronger fields, still more complicated phenomena can occur. For example, as the vector field $\mathbf{p}(\mathbf{q}, t)$ oscillates, a caustic in the extended space could pass through the point $\left(\mathbf{q}=0, E=E_{\text {out }}\right)$. This would be a bifurcation, at which the number of closed orbits satisfying the energy condition (5.1) changes; equivalently, the number of extrema of $S(t)$ changes. Such phenomena certainly exist, but at present, significant consequences have not been identified in the experiments.

Note that the amplitude factor $A_{0}(\hat{t})\left|d E_{\text {ret }} / d t\right|^{-1 / 2}$ that appears in Eq. (5.2) is the same as the amplitude factor that enters into the semiclassical energy-domain Green's function [15], and the "sign" that appears in the exponent combines with $\lambda$ to give $\mu$, the Maslov index that appears in the Green's function.

\section{COMPARISON WITH EXPERIMENT}

\section{A. Scaled variable spectroscopy}

The classical dynamics of hydrogen in external static and microwave electric fields (like that of hydrogen in other external field configurations) is scalable. This means that it is possible to change the external parameters in a certain way that leaves the classical dynamics unchanged except for an overall scale. The atomic core does not have this scaling property, in principle ruining the scaling property for nonhydrogenic atoms. However, in practice the effect of the core is small enough that the scaling law is still an excellent approximation.

We denote scaled quantities with tildes. We opt to make the scaled static electric field strength equal to unity: $\widetilde{F}_{0}$ $\equiv 1$. This choice dictates how the other scaled variables are defined

$$
\begin{aligned}
& \tilde{\mathbf{r}}=\mathbf{r} F_{0}^{1 / 2}, \\
& \tilde{\mathbf{p}}=\mathbf{p} F_{0}^{-1 / 4}, \\
& \tilde{t}=t F_{0}^{3 / 4}, \\
& \tilde{H}=H F_{0}^{-1 / 2}, \\
& \tilde{\omega}=\omega F_{0}^{-3 / 4} .
\end{aligned}
$$

We define the scaled rf electric field

$$
f \equiv \widetilde{F}_{1}=F_{1} / F_{0},
$$

ending up with the following final scaled Hamiltonian:

$$
\widetilde{H}=\frac{1}{2} \widetilde{\mathbf{p}}^{2}-\frac{1}{\tilde{r}}+\widetilde{z}[1+f \sin (\tilde{\omega} \widetilde{t})] .
$$

In the oscillating field, the electron energy $E(t)$ is not constant. But its initial energy $E_{\text {out }}$ is well defined, so we define the scaled energy

$$
\epsilon \equiv E_{\text {out }} F_{0}^{-1 / 2} \text {. }
$$

Action has units of momentum times distance, so the scaled action is

$$
\widetilde{S}=F_{0}^{1 / 4} S .
$$

The analogous quantum system is not scalable. Quantum mechanics imposes another relationship between the variables, namely, the commutation relation $[x, p]=i \hbar$. Effectively quantum mechanics introduces an additional scale- the de Broglie wavelength of the electron-into the system, breaking the scaling property. Specifically, under the above scaling, the classical orbits do not change their shapes but they do change their sizes compared to the de Broglie wavelength of the electron. After scaling, Planck's constant has the effective value $1 / \hbar_{\mathrm{eff}} \equiv w \equiv F_{0}^{-1 / 4}$, because

$$
S\left(E_{\text {out }}, F_{0}, F_{1}, \omega\right) / \hbar=\widetilde{S}(\epsilon, f, \tilde{\omega}) w .
$$

This scaling property allows a useful experimental technique called scaled-variable spectroscopy. To obtain a scaled spectrum, one simultaneously varies the laser energy, static, and rf field strengths, and the rf frequency to keep the scaled parameters $(\epsilon, f$, and $\tilde{\omega})$ constant while changing $F_{0}$. One records the photoabsorption spectrum as a function of $w=F_{0}^{-1 / 4}$. This technique keeps classical dynamics constant while varying the effective Planck's constant, thereby simplifying semiclassical mechanics. Then one takes the Fourier transform of the experimental scaled spectrum to obtain a "recurrence spectrum" $C(\widetilde{S})$,

$C(\widetilde{S}) \equiv\left|\frac{1}{w_{2}-w_{1}} \int_{w_{1}}^{w_{2}} d w \bar{R}_{x}\left(\epsilon, f, \tilde{\omega}, F_{0}=w^{-4}\right) \exp [-i \widetilde{S} w]\right|^{2}$,

which, because each orbit causes a sinusoidal modulation of the absorption rate, shows peaks at the scaled actions of the important classical electron orbits.

\section{B. Many orbits are similar to the parallel orbit}

In this section we will consider some of the special features of hydrogen in a static electric field, and their ramifications for the experiment. The system is classically regular, and the $\phi$ motion is ignorable. Closed orbits can be characterized by two integers, $k$ and $n$. The closed orbit $(k, n)$ undergoes $n$ cycles of $z$ motion, while undergoing $k$ cycles of transverse motion. Closed orbits return to the atom at the same angle they departed, $\theta_{f}=\theta_{i}$. Moreover, whenever the electron returns exactly to the atom, the Coulomb force turns it around and sends it back out in the direction from which it came. Therefore, in static fields, after each traversal of a closed orbit, the electron goes back out and traverses the same orbit in reverse-i.e., closed orbits repeat themselves to form longer closed orbits. 


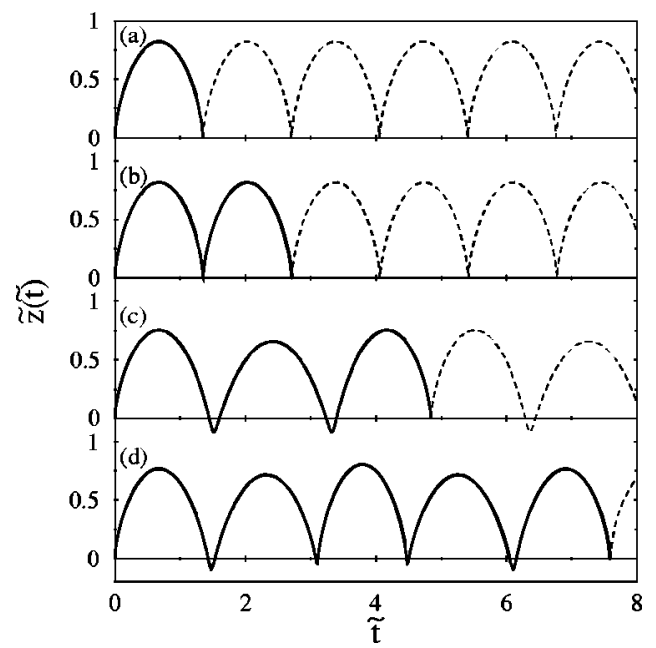

FIG. 4. Comparison of the $z$ motion of several closed orbits in static fields, with $\epsilon=-0.4$. (a) The parallel orbit $(k, n)=(0,1)$; the (b) $(1,2),(c)(1,3)$, and (d) $(2,5)$ orbits (solid lines). Repeats of the orbits are shown (dashed lines) to aid comparison. Both axes are unitless scaled quantities [see Eq. (6.1)].

One important closed orbit is the "parallel" orbit, in which the electron travels along the positive $z$ axis with no transverse motion. The parallel orbit is characterized by indices $(k, n)=(0,1)$, which we will abbreviate as $\|$. The parallel orbit repeated $n$ times has indices $(0, n)$.

At the energy and field strengths involved in the Spellmeyer-Kleppner experiment [9], the parallel orbit is particularly important. At their scaled energy $\epsilon=-0.4$, the largest possible initial angle $\theta_{i}$ of any closed orbit is about $30^{\circ}$; orbits with larger initial angles ionize immediately. Moreover, the motions along the $z$ axis are larger than those transverse to it. It turns out that the $z$ motion of any closed orbit $(k, n)$ is not so different from that of the parallel orbit repeated $n$ times (see Fig. 4):

$$
z_{k, n}(\tau) \approx z_{0, n}(\tau): 0 \leqslant \tau \leqslant T_{k, n}
$$

For example, the return time of each orbit varies by only about $30 \%$ from the return time of the repeated parallel orbit: $T_{k, n} \approx T_{0, n}=n T_{\|}$. Moreover, the actions of all orbits with the same $n$ are indistinguishable in the experiment: $S_{k, n} \approx S_{0, n}$ $=n S_{\|}$. The ac dipole moment of orbit $(k, n)$ is, accordingly [from Eq. (6.3)],

$$
Z_{k, n}(\omega) \approx Z_{0, n}(\omega)
$$

We can compute $Z_{0, n}(\omega)$ for $n$ repetitions of the parallel orbit from the ac dipole moment $Z_{\|}(\omega)$ of the parallel orbit itself as follows:

$$
\begin{aligned}
Z_{0, n}(\omega)= & \frac{1}{T_{0, n}}\left[\int_{0}^{T_{\|}}+\int_{T_{\|}}^{2 T_{\|}}+\cdots+\int_{(n-1) T_{\|}}^{n T_{\|}}\right] z_{0, n}(\tau) \\
& \times \exp (i \omega \tau) d \tau \\
= & \frac{1}{n T_{\|}}\left[1+e^{i \omega T_{\|}}+\cdots+e^{i \omega(n-1) T_{\|}}\right]
\end{aligned}
$$

$$
\begin{aligned}
& \times \int_{0}^{T_{\|}} z_{\|}(\tau) \exp (i \omega \tau) d \tau \\
= & \exp \left[\frac{i \omega(n-1) T_{\|}}{2}\right] \frac{\sin \left(n \omega T_{\|} / 2\right)}{n \sin \left(\omega T_{\|} / 2\right)} Z_{\|}(\omega)
\end{aligned}
$$

(A similar expression relates the ac dipole moment of any repeated orbit to that of its primitive suborbit.) Substituting Eqs. (6.4) and (6.5) into Eq. (4.6), we find that all recurrences $(k, n)$ close to the $n$th return of the parallel orbit are reduced by approximately the same factor,

$$
J_{0}\left(e F_{1}\left|Z_{k, n}(\omega)\right| T_{k, n} / \hbar\right) \approx J_{0}\left(\frac{e}{\hbar} F_{1}\left|Z_{\|}(\omega)\right| T_{\|} \frac{\sin \left(n \omega T_{\|} / 2\right)}{\sin \left(\omega T_{\|} / 2\right)}\right) .
$$

Whenever the quantity multiplying $F_{1}$ in the argument of the Bessel function becomes zero, the recurrence will not be weakened even for quite large rf fields. This happens when $Z_{\|}(\omega)$ vanishes, but also for combinations of the orbital period and rf frequency for which $\sin \left(n \omega T_{\|} / 2\right) / \sin \left(\omega T_{\|} / 2\right)$ vanishes.

For example, consider rf periods longer than the period of the parallel orbit, $T_{\mathrm{rf}}>T_{\|}$(as in the experiment). Then the sine in the denominator of Eq. (6.6) cannot vanish, but the one in the numerator vanishes if $T_{\mathrm{rf}}=(\mathrm{n} / \mathrm{m}) T_{\|}$for any integer $m$. In particular, if $T_{\mathrm{rf}}=n T_{\|}$, then recurrences near the $n$th return of the parallel orbit are unweakened by the oscillating field. At first glance, this result might seem unexpected. Normally one finds that an orbit is most perturbed when the period of the perturbation is rationally related to the period of the orbit. In our case the perturbation is a pure sinusoid, so it has no higher harmonics. Therefore, the effect of the rf field averages to zero over the multiple periods of the electron orbit, leaving the recurrence unaffected by the rf field for many rational ratios of periods.

This pattern of unweakened recurrences is the most striking feature of the experimental measurements. The "restricted" model described in this section, using only the properties of the parallel orbit, is able to predict that pattern.

\section{Experiment}

In Ref. [9], we compared two versions of the theory with experiments. The first, "general" semiclassical result comes from finding each classical closed orbit, its amplitude, and its ac dipole moment $Z(\omega)$ individually, then summing them together coherently, using a term like Eq. (4.6) for each orbit. The general theory gives quantitative agreement with the finer details of the experiment and of a semiquantal calculation [9].

The second, "restricted" semiclassical result comes from treating all orbits as approximated by the parallel orbit, as explained in Sec. VIB. The whole rf dependence of the recurrence spectrum can then be derived from the properties of just the parallel orbit. Here we give a more detailed comparison of the semiclassical theory with experiments (Fig. 5).

Since the "restricted" theory suffices to explain all the major trends in the data, we use it in the comparison. One 

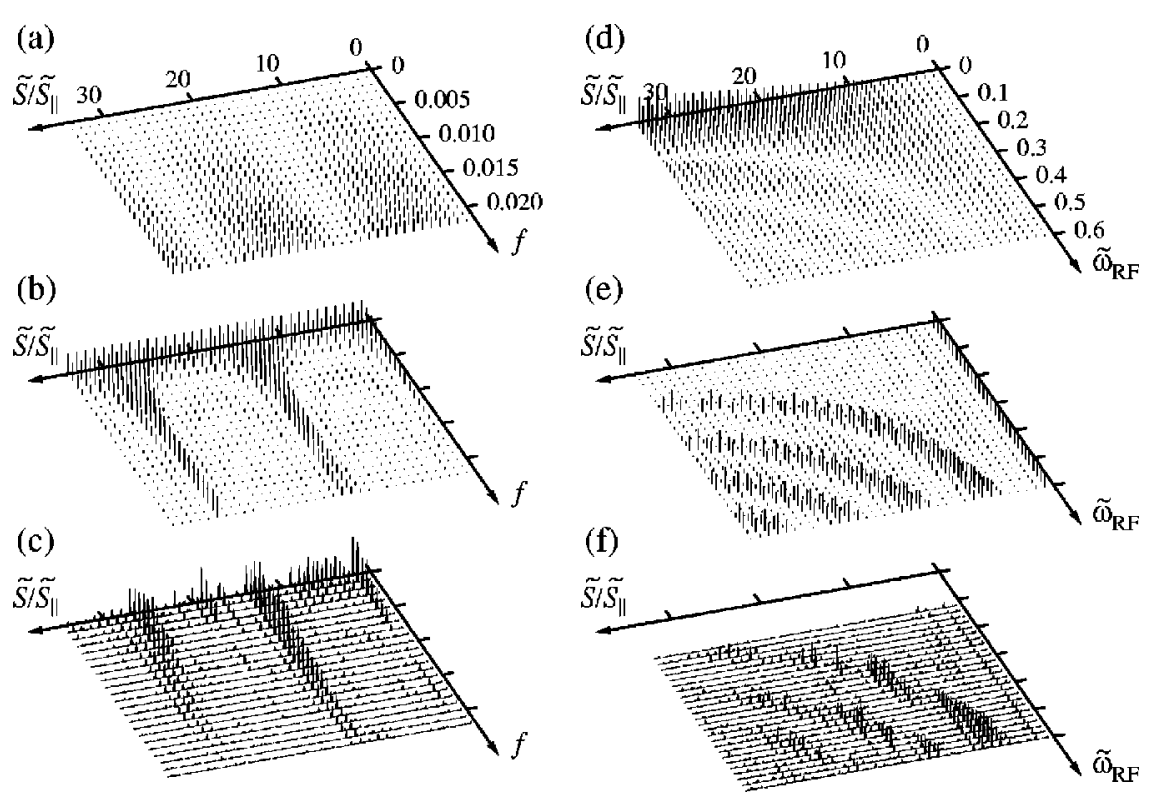

FIG. 5. Semiclassical theory vs experimental data at scaled energy $\epsilon=-0.4$. Axes are plotted as unitless scaled quantities. (a) The argument of the Bessel function given in Eq. (6.6), plotted as a function of scaled action $\widetilde{S} / \widetilde{S}_{\|}$and scaled $\mathrm{rf}$ field strength $f$. The axis $\widetilde{S} / \widetilde{S}_{\|} \approx n$ corresponds to the number of repetitions of the parallel orbit. (b) The square of the Bessel function (6.6), as a function of the same parameters. (c) Experimental recurrence spectra at various values of $f$. Parts (a), (b), and (c) are taken at a fixed scaled rf frequency $\tilde{\omega}=0.32$. Parts (d), (e), and (f) show analogous quantities, except with fixed scaled rf field strength $f=0.008$, and a range of scaled $\mathrm{rf}$ frequencies $\tilde{\omega}$. The "restricted" semiclassical theory is seen to predict the pattern of weak and strong recurrences seen in the experiment. The physical parameter ranges in each case were $146 \leqslant w \leqslant 158$; i.e., $-4.1 \quad \mathrm{~cm}^{-1}$ $\leqslant E_{\text {out }} \leqslant-3.5 \mathrm{~cm}^{-1}$ and $11.4 \mathrm{~V} / \mathrm{cm} \geqslant F_{0}$ $\geqslant 8.2 \mathrm{~V} / \mathrm{cm}$. (The experimental data are from Spellmeyer and Kleppner [16].) abscissa in each plot in Fig. 5 is $\widetilde{S} / \widetilde{S}_{\|}$, which counts the approximate number of vertical excursions that the orbit takes before returning to the atom. For plots (a)-(c) $\omega$ is held constant and the scaled rf field strength $f$ is varied; for (d) $-(\mathrm{f})$ $f$ is held constant, and the scaled $\mathrm{rf}$ frequency $\tilde{\omega}$ is varied.

Figures 5(a) and 5(d) show the argument of the Bessel function of Eq. (6.6). When that quantity goes to zero, the corresponding recurrence is not significantly weakened by the rf field. Parts (b) and (e) show the square of the Bessel function shown in Eq. (6.6), which is the factor by which recurrences are weakened by the rf field; i.e., the strength of the $f=0$ recurrence peak is multiplied by this factor. (In the experiment $\Delta w \ll w$, so the Bessel function can be treated as approximately constant across the spectrum.) Parts (c) and (f) show experimental recurrence spectra [see Eq. (6.2)] measured by Spellmeyer and Kleppner [16]. It is evident that even this restricted theory gives remarkable agreement with experiment: the unweakened bands and all of the ripply structure of the Bessel function are manifested in the experimental measurements.

\section{SYMMETRY-BREAKING ANALOGY}

We conclude this paper by defining "temporal symmetry breaking," and explaining how that concept connects the present work with other recent work. In the static field, the Hamiltonian is independent of time, while in the perturbing rf field it is periodic in time. We compare this to another system [17], an atom in a static magnetic field, which is perturbed by a weak electric field perpendicular to the magnetic field. In that case, the unperturbed Hamiltonian is independent of the azimuthal angle, while the full Hamiltonian is periodic in that angle-we call it cylindrical symmetry breaking. In this section we explain the intimate relationship between the present case of temporal symmetry breaking and the case of cylindrical symmetry breaking.

This connection came as a surprise. In quantum mechanics time plays a distinct role as an evolution parameter, not a dynamical variable, so it is not in general analogous to a coordinate or angle. On the other hand, in classical mechanics we can extend the phase space from $(\mathbf{p}, \mathbf{q})$ to $(\mathbf{p}, \mathbf{q}, t$, $-E)$, and then time becomes a dynamical variable like any

TABLE II. Correspondence between cylindrical and temporal symmetry breaking.

\begin{tabular}{lcccc}
\hline \hline & (a) & $\left(\mathrm{a}^{\prime}\right)$ & $(\mathrm{b})$ & $\left(\mathrm{b}^{\prime}\right)$ \\
\hline$H_{0}$ & $H_{\text {atomic }}+$ & $H_{\text {atomic }}+$ & $H_{\text {atomic }}+F z$ & $H_{\text {atomic }}+F z$ \\
$L_{z} B+B^{2} \rho^{2} / 8$ & $L_{z} B+B^{2} \rho^{2} / 8$ & 0 & $F_{1} z \cos (\omega t)$ \\
$H^{\prime}$ & 0 & $F_{1} x=F_{1} \rho \cos \phi$ & $L_{z}$ only \\
Conserved & $E$ and $L_{z}$ & $E$ only & $E$ and $L_{z}$ & $S_{0}-F_{1} T|Z(\omega)| \cos (\omega t)$ \\
Classical action & $S_{0}(E, B)$ & $S_{0}-F_{1} T \bar{x} \cos \left(\phi-\phi_{0}\right)$ & $S_{0}(E, F)$ & $J_{0}\left(F_{1} T|Z(\omega)| / \hbar\right)$ \\
Recurrence weakened by & - & $J_{0}\left(F_{1} T \bar{x} / \hbar\right)$ & - & {$[9]$} \\
Reference & {$[1]$} & {$[17]$} & {$[3]$} & \\
\hline \hline
\end{tabular}


other. Therefore a classical Hamiltonian that is periodic in time is analogous to one that is periodic in an angle. Within a semiclassical approximation, then, we can exploit this analogy, and connect the observations made in [9] to the observations of cylindrical symmetry breaking presented in Ref. [17]. Four closely related systems are listed in Table II.

For each of these systems, the Hamiltonian is dominated by a time-independent and cylindrically symmetric part, which we call $H_{0}$; it consists of the atomic Hamiltonian [Eq. (1.1a)] plus a term from the strong static field. In (a) and $\left(\mathrm{a}^{\prime}\right)$, the static magnetic field gives the additional term $L_{z} B+B^{2} \rho^{2} / 8$. In (b) and $\left(\mathrm{b}^{\prime}\right)$, the static electric field gives the additional term $F_{1} z$.

\section{A. Cylindrical symmetry breaking}

In the magnetic field, the simplest orbit, which produces one of the strongest recurrences, lies in the plane $z=0$; it is known as the perpendicular, Garton-Tomkins-Edmonds, or quasi-Landau orbit. Actually it is a cylindrical family of orbits; the electron begins at any initial azimuthal angle, and as it executes one cycle of $\rho$ motion, the azimuthal angle advances approximately $2 \pi / 3 \mathrm{rad}$. The electron begins with a certain energy $E_{\text {out }}$ and with angular momentum component $L_{z}^{\text {out }}=0$, both of which are conserved under $H_{0}$. It returns therefore with $L_{z}^{\mathrm{ret}}=0$, so every orbit in the cylindrical family comes back exactly to the nucleus. The cylindrical family of returning orbits carries a well-focused, cylindrically symmetric returning wave, which produces a strong recurrence.

Any interaction that changes the angular momentum $L_{z}$ weakens the recurrence. Let us turn to situation $\left(a^{\prime}\right)$. The weak electric field breaks the cylindrical symmetry, and the Hamiltonian becomes periodic in $\phi$. The cylindrical family of returning orbits is destroyed - of that continuous family, only two discrete orbits return exactly to the nucleus. All the other orbits in the family return with $L_{z}$ small but nonzero, and they miss the nucleus. They come back close enough that they contribute to the recurrence, but they do not come back in phase. This partially destructive interference weakens the recurrence.

The symmetry breaking perturbation changes the phase of the returning wave according to the formula

$$
\begin{gathered}
\frac{\partial S}{\partial F_{1}}=-\int \frac{\partial H}{\partial F_{1}} d t, \\
\Delta S=-e F_{1} \int x(t) d t,
\end{gathered}
$$

where $x(t)$ is evaluated with $F_{1}=0$. Since unperturbed orbits in a family are identical except for their azimuthal orientation, it is easy to show that the above integral depends sinusoidally on the orbit's initial azimuthal angle $\phi$, specifically,

$$
S\left(E, B ; F_{1}, \phi\right) \approx S_{0}(E, B)-F_{1} T \bar{x} \cos \left(\phi-\phi_{0}\right) .
$$

[Here $\bar{x}$ is the average $x$ coordinate of the "most uphill" orbit in the family, $\bar{x}=\max \int x(t) d t / T$, and $\phi_{0}$ is the initial azimuthal angle of that orbit.]

The returning wave function at the origin is the coherent superposition of all the waves that return after starting out at all initial azimuths. Therefore, the recurrence amplitude is also such a superposition:

$$
\begin{aligned}
R_{x}= & \operatorname{Im} \frac{1}{2 \pi} \int_{0}^{2 \pi} d \phi C \exp \left\{i\left[S\left(E, B ; F_{1}, \phi\right) / \hbar+\gamma\right]\right\} \\
\approx & \operatorname{Im}\left\{C \exp \left\{i\left[S_{0}(E, B) / \hbar+\gamma\right]\right\} \frac{1}{2 \pi} \int_{0}^{2 \pi} d \phi\right. \\
& \left.\times \exp \left[-(i / \hbar) F_{1} T \bar{x} \cos \left(\phi-\phi_{0}\right)\right]\right\} \\
= & C \sin \left[S_{0}(E, B) / \hbar+\gamma\right] J_{0}\left(F_{1} T \bar{x} / \hbar\right) .
\end{aligned}
$$

From this formula, we see that partially destructive interference of waves coming from different azimuths weakens the recurrence amplitude by a Bessel function factor. The argument of the Bessel function is proportional to the perturbing electric field $F_{1}$, and to the product of the time duration of the unperturbed orbit $T$ with the average of $x(t)$ on the mostuphill unperturbed orbit, i.e., to the static electric dipole moment of that orbit.

The $\phi$ integral in Eq. (7.3) has two stationary phase points, corresponding to two terms in the asymptotic approximation for the Bessel function. These correspond to two surviving closed orbits. Therefore, Eq. (7.3) smoothly connects the cylindrically symmetric case with the case of fully broken symmetry.

\section{B. Temporal symmetry breaking}

Now let us return to the case considered in this paper, (b) and $\left(\mathrm{b}^{\prime}\right) . H_{0}$ contains the Coulomb Hamiltonian with a static electric field along the $z$ axis, and the perturbation $H^{\prime}$ is a weak oscillating electric field also oriented along the $z$ axis. As explained earlier, because of the intrinsic time dependence of this system, many aspects of recurrence theory had to be re-examined. First we had to show that an excitation rate or photon absorption rate exists in this system [Eq. (2.7)]: at each time $t$, there is a well-defined photoabsorption cross section and an oscillator strength density $D f\left(E, F_{0} ; F_{1}, t\right)$, which oscillates at the rf frequency. Not surprisingly, this quantity was divided into a smooth "background" part and an oscillatory part associated with returning waves [Eqs. (2.15) and (3.9a)].

The returning waves were, as always, constructed from the classical orbits. At this point, we went into the extended phase space $(\mathbf{p}, \mathbf{q}, t,-E)$, and then time became a dynamical variable like any other [Eq. (B3)]. From this point on, the theory exactly parallels the theory of cylindrical symmetry breaking [17].

Since $H(t)$ is periodic in time, time is like an angle variable; it is analogous to the azimuth $\phi$. Each returning orbit for static fields becomes redefined as a continuous family of 
orbits returning at time $t$ [Eq. (3.6)], and we examine this family over one cycle of the $\mathrm{rf}$ field. Because of the $t$-symmetry breaking, the conjugate variable $E$ is not conserved. The family of orbits that formerly came back to the nucleus with $E_{\text {ret }}=E_{\text {out }}$ is destroyed. Of that continuous family of unperturbed orbits, under sufficiently small perturbation, the whole family will remain closed in configuration space, but not closed in the extended space $(\mathbf{q},-E)$. In each family, just two discrete orbits survive that have $E_{\text {ret }}=E_{\text {out }}$.

Recurrences are weaker if $E_{\text {ret }} \neq E_{\text {out }}$. (This important fact was not obvious from the earlier derivations of recurrence theory, in which energy conservation was taken for granted $[1,3]$.) The situation is analogous to cylindrical symmetry breaking, in which recurrences are weaker if angular momentum is not conserved. If the oscillating field is sufficiently strong, then only the two distinct orbits for which $E_{\text {ret }}=E_{\text {out }}$ contribute to the recurrence. On the other hand, for a weak perturbation, the whole family contributes, but with a time-varying phase.

The phase of the returning wave is the extended action, and the first-order calculation gives

$$
\Delta S(t)=-e F_{1} T|Z(\omega)| \sin (\omega t+\beta) .
$$

Instead of the static dipole moment $\bar{x}, \Delta S$ depends on the ac-dipole moment $Z(\omega)$. The contribution to the excitation rate arising from each recurrence is

$$
R_{x}\left(E, F_{0} ; F_{1}, t\right)=C \sin \left[S\left(E, F_{0} ; F_{1}, t\right) / \hbar+\gamma\right]
$$

which, when averaged over a cycle, gave the Bessel function,

$$
\bar{R}_{x}\left(E, F_{0} ; F_{1}\right)=C \sin \left[S_{0}\left(E, F_{0}\right) / \hbar+\gamma\right] J_{0}\left[F_{1} T|Z(\omega)| / \hbar\right] .
$$

Partially constructive averaging of the recurrence term over a cycle of the RF field selectively reduces the strengths of the recurrences. For certain recurrences, generally those having return times commensurable with $T_{\text {rf }}$, the ac dipole moment vanishes, and the recurrence survives the perturbation.

\section{ACKNOWLEDGMENTS}

This research was supported by the National Science Foundation and the Office of Naval Research through grants to the College of William and Mary. We thank N. Spellmeyer and D. Kleppner for initiating the experiments that led to these theoretical developments.

\section{APPENDIX A: PROOFS AND ADDITIONAL FORMULAS}

\section{Absorption rate, cross section, and oscillator strength density}

For comparison to earlier work, we note that the photoabsorption cross section is equal to the rate of production of electrons in excited levels $R_{x}(t)$, divided by the photon flux density in the laser:

$$
\begin{aligned}
\sigma(t) & =\frac{R_{x}(t)}{c\langle\mathbf{E} \times \mathbf{H}\rangle /\left(4 \pi \hbar \omega_{L}\right)} \\
& =\frac{4 \pi \hbar \omega_{L} R_{x}(t)}{c\left\langle 4 F_{L}^{2} \cos ^{2}\left(\omega_{L} T+\gamma_{L}\right)\right\rangle} \\
& =\frac{2 \pi \hbar \omega_{L}}{c F_{L}^{2}} R_{x}(t) .
\end{aligned}
$$

In the last line we have averaged over a cycle of the laser field. The oscillator strength density can be defined as

$$
D f(t)=\frac{m_{e} c}{2 \pi^{2} e^{2} \hbar} \sigma(t)=\frac{m_{e} \omega_{L}}{\pi e^{2} F_{L}^{2}} R_{x}(t) .
$$

Its dimensions are (energy) $)^{-1}$. The cross section and the oscillator strength density, like the absorption rate, are time dependent, and must be averaged over a cycle of the rf field (Sec. IV B). These formulas are based on Gaussian units for electric fields and charges.

\section{Proof of Eq. (2.7)}

The rate of increase of probability of finding the electron in the excited state is given by Eq. (1.5), which can be written

$$
R_{x}(t)=2 \operatorname{Re}\left\{\left[\frac{d}{d t}\left\langle\Psi_{x}(t)\right|\right]\left|\Psi_{x}(t)\right\rangle\right\} .
$$

From Eq. (2.3),

$$
\frac{d}{d t}\left\langle\Psi_{x}(t)\right|=(-i \hbar)^{-1}\left[\langle I(t)|+\left\langle\Psi_{x}(t)\right| H(t)\right] .
$$

Therefore,

$$
R_{x}(t)=(2 / \hbar) \operatorname{Re}\left[i\left\langle I(t) \mid \Psi_{x}(t)\right\rangle+i\left\langle\Psi_{x}(t)|H(t)| \Psi_{x}(t)\right\rangle\right] .
$$

The second term in the brackets is purely imaginary, leaving

$$
\begin{aligned}
R_{x}(t) & =-(2 / \hbar) \operatorname{Im}\left\langle I(t) \mid \Psi_{x}(t)\right\rangle \\
& =\left(2 / \hbar^{2}\right) \operatorname{Re} \int_{-\infty}^{t} d t^{\prime}\left\langle I(t)\left|K^{+}\left(t, t^{\prime}\right)\right| I\left(t^{\prime}\right)\right\rangle .
\end{aligned}
$$

In the last equation we used Eq. (2.6). Equation (A3) is equivalent to Eq. (2.16a) of Ref. [1].

\section{Outgoing Coulomb wave $\psi_{\text {out }}(q)$}

In this appendix we justify Eq. (2.12) and give an expression for computing $\mathcal{Y}(\theta, \phi)$. More details can be found in Ref. [1(b)], Sec. IV C.

We compute $\psi_{\text {out }}$ from Eq. (2.11). For this we need the expression for the Coulomb Green's function. Since the experiments are near the ionization threshold, we use the zeroenergy Green's function, which is accurate enough for our purposes: 


$$
G_{C}^{+}\left(\mathbf{q}, \mathbf{q}^{\prime} ; E=0\right)=\sum_{l, m} Y_{l m}(\theta, \phi) g_{l}^{0}\left(r, r^{\prime}\right) Y_{l m}^{*}\left(\theta^{\prime}, \phi^{\prime}\right),
$$

where, when $r>r^{\prime}$,

$$
g_{l}^{0}\left(r, r^{\prime}\right)=-2 \pi i \frac{H_{2 l+1}^{(1)}(\sqrt{8 r})}{\sqrt{r}} \frac{J_{2 l+1}\left(\sqrt{8 r^{\prime}}\right)}{\sqrt{r^{\prime}}} .
$$

$J_{2 l+1}$ is a Bessel function; $H_{2 l+1}^{(1)}$ is a Hankel function of the first kind, for which we use the asymptotic expansion

$$
H_{\nu}^{(1)}(z) \stackrel{z \gg 1}{\rightarrow} \sqrt{\frac{2}{\pi z}} \exp [i(z-\nu \pi / 2-\pi / 4)] .
$$

Substitute Eqs. (A4), (A5), and (A6) into Eq. (2.11), and compare with Eq. (2.12). Taking the constant $C_{1}$ equal to

$$
C_{1}=2^{3 / 4} \pi^{1 / 2} e^{-i 5 \pi / 4} \quad\left(m_{e} a_{0}^{2} / \hbar^{2}\right),
$$

the angular distribution of outgoing waves is found to be

$$
\begin{aligned}
\mathcal{Y}(\theta, \phi)= & \sum_{l, m}(-)^{l} Y_{l m}(\theta, \phi) \int d \mathbf{q}^{\prime} Y_{l m}^{*}\left(\theta^{\prime}, \phi^{\prime}\right) \\
& \times \frac{J_{2 l+1}\left(\sqrt{8 r^{\prime}}\right)}{\sqrt{r^{\prime}}} D_{z} \psi_{i}\left(\mathbf{q}^{\prime}\right) .
\end{aligned}
$$

$D_{z}$ is the dipole operator appropriate for the laser polarization (in our case $D_{z}=z^{\prime}=r^{\prime} \cos \theta^{\prime}$ ). The angular integral in Eq. (A8) is standard, and the radial integral can be evaluated as described in Ref. [1]. It can be shown that Eq. (A8) is equivalent to Eq. (2.13), if $C_{2}$ is chosen to be

$$
C_{2}=2^{3 / 2} \pi \quad\left(m_{e} a_{0}^{3} / \hbar^{2}\right) .
$$

\section{Returning wave}

The goal of this section is to evaluate the factor $N(t)$, which characterizes the strength of $\Psi_{x}^{\text {ret }}$ in Eq. (3.6). We do this by computing the ratio of the incoming part of $\Psi_{x}^{\mathrm{sc}}$ to the incoming part of $\psi_{C, \theta_{f}}$ at the convenient position $\left(r_{f}, \theta_{f}\right)$,

$$
N(t)=\frac{\operatorname{inc}\left[\Psi_{x}^{\mathrm{sc}}\left(r_{f}, \theta_{f}, t\right)\right]}{\operatorname{inc}\left[\psi_{C, \theta_{f}}\left(r_{f}, \theta_{f}\right)\right]}
$$

where "inc" means "incoming part of."

Except near a bifurcation, these two approximations are consistent, and $N(t)$ is approximately independent of $r$ in a shell (say from $10 a_{0}$ to $70 a_{0}$ ) around the nucleus. Thus the final answer is insensitive to the radius chosen to "join" the semiclassical wave to the Coulomb scattering wave.

The incoming part of $\psi_{C, \theta_{f}}(r, \theta)$ at the angle $\theta_{f}$ (the direction from which the returning wave comes) on a final boundary sphere of radius $r_{f}$ is

$$
\operatorname{inc} \psi_{C, \theta_{f}}\left(r_{f}, \theta_{f}\right) \approx C_{3} \frac{\exp \left(-i \sqrt{8 r_{f}}\right)}{r_{f}^{1 / 2} \sin \theta_{f}}
$$

where

$$
C_{3}=e^{i \pi / 2} 2^{-3 / 2} \pi^{-1} \quad\left(m_{e} a_{0}^{3} / \hbar^{2}\right) .
$$

[This is Eq. (4.23a) of Ref. [1(b)], evaluated at $\theta=\theta_{f}$.]

Now the coefficient $N(t)$ can be evaluated from Eq. (A10) using Eqs. (3.1), (A11a), (2.10), and (2.12):

$$
\begin{aligned}
N(t)= & e F_{L} \frac{C_{1}}{C_{3}}\left[\frac{r_{f}^{1 / 2}}{r_{i}^{3 / 4}} A\left(\mathbf{q}_{f}, t ; \mathbf{q}_{i}, t_{i}\right) \sin \theta_{f}\right] \mathcal{Y}\left(\theta_{i}\right) \\
& \times \exp \left\{i\left[\mathcal{S}\left(\mathbf{q}_{f}, t ; \mathbf{q}_{i}, t_{i}\right)+\sqrt{8 r_{i}}+\sqrt{8 r_{f}}-E_{\text {out }} t\right] / \hbar\right. \\
& -i \lambda \pi / 2\}
\end{aligned}
$$

As stated earlier, as long as we are not near a bifurcation, our approximations are all consistent and the value of $N(t)$ is independent of the location of the boundary sphere. In fact, the limits can be evaluated carefully to compute $N(t)$ at $r_{i}$ $=r_{f}=0$. In particular, the combination

$$
\lim _{r_{f}, r_{i} \rightarrow 0}\left[\mathcal{S}\left(\mathbf{q}_{f}, t ; \mathbf{q}_{i}, t_{i}\right)+\sqrt{8 r_{i}}+\sqrt{8 r_{f}}\right]=\mathcal{S}\left(\mathbf{0}, t ; \mathbf{0}, t_{i}\right)
$$

is equal to the classical action $\mathcal{S}\left(\mathbf{0}, t ; \mathbf{0}, t_{i}\right)$ calculated from origin to origin, while the combination

$$
\lim _{r_{f}, r_{i} \rightarrow 0}\left[\left(r_{f}^{1 / 2} / r_{i}^{3 / 4}\right) A\left(\mathbf{q}_{f}, t ; \mathbf{q}_{i}, t_{i}\right) \sin \theta_{f}\right] \equiv A_{0}(t)
$$

has a finite limit, which we call $A_{0}(t)$. Substituting those quantities into Eq. (A12), we obtain Eq. (3.7). Equation (3.9c) gives an expression for $A_{0}(t)$ in terms of reduced Jacobians.

\section{Excitation rate from returning orbits}

Using the definitions of $\psi_{C, \theta_{f}}$ [Eq. (3.5)] and of $\mathcal{Y}(\theta)[\mathrm{Eq}$. (2.13) or (A8)], one easily shows that

$$
\left\langle D_{z} \psi_{i} \mid \psi_{C, \theta_{f}}\right\rangle=C_{2} \mathcal{Y}^{*}\left(\theta_{f}\right)
$$

where $C_{2}$ is defined in Eq. (A9). The overlap of the returning wave with $D_{z} \psi_{i}$ gives the contribution of that wave to the excitation rate $R_{x}^{\text {ret }}(t)$. Starting from Eq. (3.8), we have only a long sequence of substitutions. In order we use Eqs. (3.6) and (2.4), then Eqs. (A15) and (3.7),

$$
\begin{aligned}
R_{x}^{\mathrm{ret}}(t)= & -(2 / \hbar)\left(e F_{L}\right) \operatorname{Im}\left[N(t) e^{i E_{\text {out }} t / \hbar}\left\langle D_{z} \psi_{i} \mid \psi_{C, \theta_{f}}\right\rangle\right] \\
= & -(2 / \hbar)\left(e F_{L}\right)^{2} \operatorname{Im}\left[\left(C_{1} C_{2} / C_{3}\right) \mathcal{Y}\left(\theta_{f}\right) \mathcal{Y}^{*}\left(\theta_{i}\right)\right. \\
& \left.\times A_{0}(t) \exp \{i S(t) / \hbar-i \lambda \pi / 2\}\right],
\end{aligned}
$$

where $S(t)=\mathcal{S}\left(\mathbf{0}, t ; \mathbf{0}, t_{i}\right)+E_{\text {out }}\left(t-t_{i}\right)$, as in Eq. (3.9b). Substituting in the values of the constants from Eqs. (A7), (A9), and (A11b) yields Eq. (3.9a).

\section{Dimensional considerations}

Let us denote dimensions of length by $l$, energy by $e$, and time by $t$. The functions $\Psi(\mathbf{q}, t), \Psi_{x}(\mathbf{q}, t)$, and $\psi_{i}(\mathbf{q})$ all 


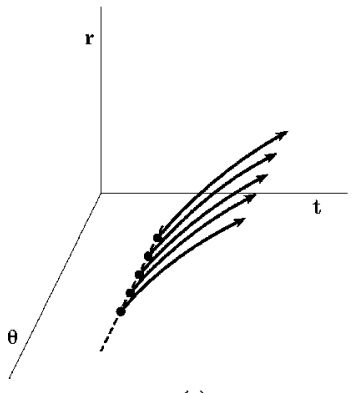

(a)

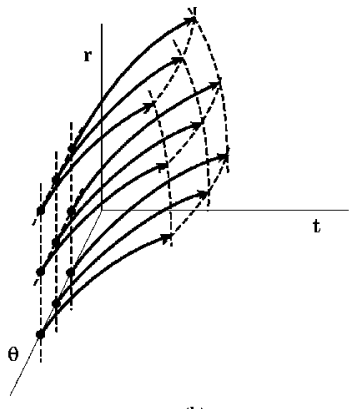

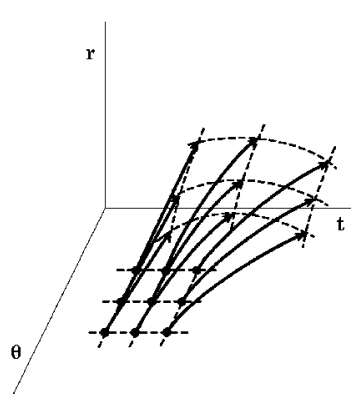

(c)

FIG. 6. (a) If the Hamiltonian is independent of time, then, as in Ref. [1], we can calculate the Lagrangian manifold (and thereby the wave function) by integrating trajectories starting at a given boundary $r=r_{i}$ at any arbitrary fixed time $t_{i}$. (b) Often in time-dependent systems, we construct a wave function $\Psi(\mathbf{q}, t)$ given its value everywhere in space at some initial time $t_{i}$. Trajectories associated with such a wave function emerge from all locations $(r, \theta)$ in the $t=t_{i}$ plane. (c) In the present case the "initial" wave function is given on a boundary $r=r_{i}$ at all times $t$. The associated trajectories emerge from all locations $(\theta, t)$ in the $r=r_{i}$ plane. The Lagrangian manifold framework naturally allows such a change.

have the usual dimensions of three-dimensional wave functions, $l^{-3 / 2}$. From Eq. (2.11), $\psi_{\text {out }}(\mathbf{q})$ has dimensions $e^{-1} l^{-1 / 2}$. The angular factor $\mathcal{Y}(\theta)$ has dimensions $l$, and it is best to take the radial part of $\psi_{\text {out }}(\mathbf{q})$ to have dimensions $l^{-3 / 2}$; then the radial factor in Eq. (2.12) would be $\exp \left[i\left(8 r / a_{0}\right)^{1 / 2}\right] /\left(r / a_{0}\right)^{3 / 4} a_{0}^{-3 / 2}$, and this means that the constant $C_{1}$ has dimensions $e^{-1}$ and its value [Eq. (A7)] is of course in Hartree ${ }^{-1}$.

In Eq. (3.5) we choose $\psi_{C, \theta_{f}}(\mathbf{q})$ to have the same dimensions as $\psi_{\text {out }}(\mathbf{q}) \quad\left(e^{-1} l^{-1 / 2}\right)$, so $N(t)$ has dimensions $(e / l)$. In Eq. (A8) let the radial factor have dimensions $l^{-3 / 2}$; then, like the radial part of $\psi_{\text {out }}$, it is $J_{2 l+1}\left(8 r / a_{0}\right)^{1 / 2} /\left(r / a_{0}\right)^{1 / 2} a_{0}^{-3 / 2}$. Similarly we interpret Eq. (A11a) to contain $\left(r_{i} / a_{0}\right)$ and $\left(r_{f} / a_{0}\right)$ everywhere, and if the radial factor in Eq. (A11a) is defined to have units $a_{0}^{-3 / 2}$, then $C_{3}$ has dimensions $(l / e)$. A similar convention is involved in Eq. (A14): with $r_{f}$ and $r_{i}$ understood as $r_{f} / a_{0}$ and $r_{i} / a_{0}$, then $A_{0}(t)$ is dimensionless, and one easily confirms that $R_{x}^{\text {ret }}(t)$ [Eq. (3.9a)] has dimensions $t^{-1}$.

\section{APPENDIX B: LAGRANGIAN MANIFOLDS AND TIME-DEPENDENT WAVE FUNCTIONS}

Outside the domain where $|I(t)\rangle$ is significant, we can construct a solution to the homogeneous time-dependent Schrödinger equation

$$
[i \hbar \partial / \partial t-H(t)] \Psi_{x}(\mathbf{q}, t)=0
$$

by the method of Maslov and Fedoriuk [14]. However, our situation is a bit different from the common one. In most applications of semiclassical methods to time-dependent problems, the initial wave function is given everywhere in space at some single initial time. In contrast, here we are constructing $\Psi(\mathbf{q}, t)$ from its behavior on a boundary sphere at all times. Nevertheless we can show that we have a valid semiclassical construction by mapping this problem onto the framework of Maslov and Fedoriuk.
For this purpose we expand phase space in the usual way by including $t$ as a classical dynamical variable, creating a conjugate momentum $p_{t}$, and defining an effective Hamiltonian

$$
\mathcal{H}\left(\mathbf{p}, \mathbf{q}, p_{t}, t\right) \equiv H(\mathbf{p}, \mathbf{q}, t)+p_{t}
$$

We create a "timelike" variable $\tau$ to represent the progress along paths in the expanded phase space, and then the canonical equations of motion are augmented by

$$
\begin{gathered}
d t / d \tau=1, \\
d p_{t} / d \tau=-\partial H / \partial t .
\end{gathered}
$$

We may construct a semiclassical wave function using the theory of Maslov and Fedoriuk, provided that we know the wave function on an "initial" two-dimensional Lagrangian manifold in the three-dimensional augmented configuration space $(r, \theta, t)$. If we were evolving a wave function in time from its known spatial dependence at a single time $t_{i}$, then that initial surface would be the set of all configuration points $(r, \theta)$ at the initial time $t_{i}$. However, as explained in the text, our initial surface is the set of points on the boundary sphere at all times-the set of all points $\left\{r=r_{i}, 0 \leqslant \theta_{i}\right.$ $\leqslant \pi,-\infty<t_{i}<\infty$ \} (see Fig. 6).

Now we must define the "generator" $S_{0}\left(r_{i}, \theta_{i}, t_{i}\right)$ and the initial momenta on this initial surface so that they are self-consistent, and consistent with $\Psi_{x}^{\mathrm{dir}}(\mathbf{q}, t)$ on the surface [Eqs. (2.10) and (2.12)]. We choose

$$
S_{0}\left(r_{i}, \theta_{i}, t_{i}\right)=-E_{\text {out }} t_{i}+\sqrt{8 r_{i}} \text {. }
$$

The second term is added for later convenience. $\mathcal{Y}(\theta)$ is a slowly varying factor which will be incorporated into the initial amplitude rather than into $S_{0}$. The corresponding initial momenta are

$$
\begin{gathered}
p_{\theta}\left(r_{i}, \theta_{i}, t_{i}\right)=\partial S_{0} / \partial \theta_{i}=0, \\
p_{t}\left(r_{i}, \theta_{i}, t_{i}\right)=\partial S_{0} / \partial t_{i}=-E_{\text {out }},
\end{gathered}
$$


while $p_{r}$ is chosen such that the value of the effective Hamiltonian $\mathcal{H}$ is fixed and equal to zero. Specification of these values of $\left(r_{i}, p_{r}, p_{\theta}, p_{t}\right)$ specifies an initial two-dimensional surface, parametrized by $\left(\theta_{i}, t_{i}\right)$, in six-dimensional phase space. The method of construction ensures that this initial manifold is Lagrangian.

Next, for each point $\left(\theta_{i}, t_{i}\right)$ on the initial surface, we integrate Hamilton's equations, generating trajectories that are functions of the progress variable $\tau$ and the initial variables $\left(\theta_{i}, t_{i}\right)$; i.e., the solutions to Hamilton's equations are represented by six functions $\left[\mathbf{q}, \mathbf{p}, p_{t}=-E(\tau)\right.$, and $\left.t=t_{i}+\tau\right]$ of three variables $\left(\theta_{i}, t_{i}\right.$, and $\left.\tau\right)$. The initial two-dimensional surface thus sweeps out a three-dimensional surface in the six-dimensional phase space, and again the method of construction ensures that it is a Lagrangian manifold. As the trajectories are integrated, they give the configuration space generator of the Lagrangian manifold, $S(\mathbf{q}, t)$ :

$$
\begin{aligned}
S(\mathbf{q}, t)= & \int \sum p_{j} d q_{j}+S_{0}\left(r_{i}, \theta_{i}, t_{i}\right) \\
= & \int_{\mathbf{q}_{i} t_{i}}^{\mathbf{q}, t}\left[p_{r} d r / d \tau+p_{\theta} d \theta / d \tau+p_{t} d t / d \tau\right] d \tau \\
& -E_{\text {out }} t_{i}+\sqrt{8 r_{i}} \\
= & \mathcal{S}\left(\mathbf{q}, t ; \mathbf{q}_{i}, t_{i}\right)-E_{\text {out }} t_{i}+\sqrt{8 r_{i}} .
\end{aligned}
$$

In this formula $\mathbf{q}_{i}=\left(r_{i}, \theta_{i}\right)$. Since $\left(\mathbf{q}_{i}, t_{i}\right)$ can be regarded as a function of the field point $(\mathbf{q}, t)$, the left-hand side is a function of $(\mathbf{q}, t)$, and it is the generator of a Lagrangian manifold having $\mathbf{p}=\partial S / \partial \mathbf{q}$, and $p_{t}=\partial S / \partial t$.

Given the wave function $\Psi_{0}$ on the initial manifold, the wave function at each point in configuration space $(\mathbf{q}, t)$ can be calculated according to the standard rules:

$$
\begin{aligned}
\Psi(\mathbf{q}, t)= & \Psi_{0}\left(\mathbf{q}_{i}, t_{i}\right) A\left(\mathbf{q}, t ; \mathbf{q}_{i}, t_{i}\right) \\
& \times \exp \left[i \mathcal{S}\left(\mathbf{q}, t ; \mathbf{q}_{i}, t_{i}\right) / \hbar-i \lambda \pi / 2\right] .
\end{aligned}
$$

In particular the phase is the generator $\mathcal{S}$ (plus the appropriate Maslov phase shifts). The amplitude is the wave function on the initial surface times a ratio of Jacobians,

$$
A\left(\mathbf{q}, t ; \mathbf{q}_{i}, t_{i}\right) \equiv\left|\frac{J\left(\mathbf{q}_{i}, t_{i}\right)}{J(\mathbf{q}, t)}\right|^{1 / 2},
$$

and each Jacobian is

$$
\begin{aligned}
J(\mathbf{q}, t) & =\operatorname{det} \frac{\partial(\text { evolving configuration space variables })}{\partial(\text { initial-surface variables; progress variable })} \\
& =\operatorname{det} \frac{\partial(x, y, z, t)}{\partial\left(\theta_{i}, \phi_{i}, t_{i} ; \tau\right)} \\
& =r^{2} \sin \theta \hat{J}(\mathbf{q}, t),
\end{aligned}
$$

where

$$
\hat{J}(\mathbf{q}, t)=\operatorname{det} \frac{\partial(r, \theta, t)}{\partial\left(\theta_{i}, t_{i} ; \tau\right)} .
$$

Since we have obeyed all the rules of Maslov and Fedoriuk, previously established theorems tell us that we have constructed the first term in a formal asymptotic approximation to a solution to the Schrödinger equation; i.e., we have a semiclassical approximation.

The relationship $t=t_{i}+\tau$ allows us to simplify Eq. (B10a). Treating the final position as a function of the initial angles, initial time, and time of travel, we obtain

$$
\hat{J}(\mathbf{q}, t)=\operatorname{det}\left[\frac{\partial(r, \theta)}{\partial\left(\theta_{i}, t_{i}\right)}\right]_{\tau}-\operatorname{det}\left[\frac{\partial(r, \theta)}{\partial\left(\theta_{i}, \tau\right)}\right]_{t_{i}},
$$

where subscripts indicate the variable that is to be held fixed. The second term of this expression is the Jacobian that was needed in Ref. [1], where the system was time independent. The first term does not appear in the time-independent theory. It includes the change in a trajectory's final position when the initial time $t_{i}$ is changed, while holding the time of flight (or travel time) $\tau$ constant. For a time-independent system, this derivative is zero. For our time-dependent system it is nonzero, because trajectories beginning at different times experience different phases of the rf field, and therefore end up at different final positions.

An alternative and more succinct expression for $\hat{J}$ is motivated by the observation that $\tau$ can be eliminated by substituting the identity

$$
\left[\frac{\partial}{\partial \tau}\right]_{t_{i}}=\left[\frac{\partial}{\partial t_{i}}\right]_{\tau}-\left[\frac{\partial}{\partial t_{i}}\right]_{t}
$$

into Eq. (B10a); the result is

$$
\hat{J}(\mathbf{q}, t)=\operatorname{det}\left[\frac{\partial(r, \theta)}{\partial\left(\theta_{i}, t_{i}\right)}\right]_{t} .
$$

In this expression, the final (laboratory) time $t$ is held fixed, while the initial time (and implicitly the travel time) are varied.

\section{APPENDIX C: ACTION PERTURBATION THEOREM}

Equation (4.2) is a variation on old theorems in classical mechanics. Its proof is a matter of writing out all the variables and taking a derivative. We are given a Hamiltonian function $H\left(\mathbf{p}, \mathbf{q}, t^{\prime} ; F_{1}\right) \equiv H\left(t^{\prime} ; F_{1}\right)$ which depends on the phase space variables $(\mathbf{p}, \mathbf{q})$, the time $t^{\prime}$, and on the strength $F_{1}$ of the rf field (though $F_{1}$ could be any other parameter). We are given a closed orbit that ends at the origin at time $t$. It begins at the origin at time

$$
t_{i}=t_{i}\left(t ; F_{1}\right)=t-T\left(t ; F_{1}\right) .
$$

$T\left(t ; F_{1}\right)$ is the duration of the closed orbit; in our case it is a periodic function of the final time $t$, and it has some unspecified dependence on the parameter $F_{1}$. The orbit is therefore described by functions $\left[\mathbf{p}\left(t^{\prime} ; t, F_{1}\right), \mathbf{q}\left(t^{\prime} ; t, F_{1}\right)\right]$ with $t_{i}\left(t, F_{1}\right) \leqslant t^{\prime} \leqslant t$.

Now we fix the final time $t$, and drop it from our list of variables. The orbit functions obey Hamilton's equations 


$$
\begin{gathered}
d \mathbf{p}\left(t^{\prime} ; F_{1}\right) / d t^{\prime}=-\partial H\left(t^{\prime} ; F_{1}\right) / \partial \mathbf{q}, \\
d \mathbf{q}\left(t^{\prime} ; F_{1}\right) / d t^{\prime}=\partial H\left(t^{\prime} ; F_{1}\right) / \partial \mathbf{p} .
\end{gathered}
$$

The end points are held fixed,

$$
\begin{gathered}
\mathbf{q}\left(t^{\prime}=t ; F_{1}\right)=\mathbf{q}_{f}, \\
\mathbf{q}\left(t^{\prime}=t_{i}\left(t, F_{1}\right) ; F_{1}\right)=\mathbf{q}_{i},
\end{gathered}
$$

and the initial momentum $\mathbf{p}_{i}=\mathbf{p}\left(t^{\prime}=t_{i}\left(F_{1}\right) ; F_{1}\right)$ is restricted such that $H\left(\mathbf{p}_{i}, \mathbf{q}_{i}, t_{i} ; F_{1}\right)=E_{\text {out }}$ is fixed and independent of $F_{1}$. Because $\mathbf{q}_{i}$ is held fixed,

$\mathbf{0}=\frac{d \mathbf{q}_{i}}{d F_{1}}=\frac{\partial \mathbf{q}\left(\mathbf{t}_{i}\left(F_{1}\right) ; F_{1}\right)}{\partial t_{i}} \frac{d t_{i}\left(F_{1}\right)}{d F_{1}}+\frac{\partial \mathbf{q}\left(t_{i}\left(F_{1}\right) ; F_{1}\right)}{\partial F_{1}}$.

The extended action $S\left(F_{1}\right)$ is

$$
\begin{aligned}
S\left(F_{1}\right)= & \int_{t_{i}\left(F_{1}\right)}^{t}\left\{\mathbf{p}\left(t^{\prime} ; F_{1}\right) \cdot \frac{\partial \mathbf{q}\left(t^{\prime} ; F_{1}\right)}{\partial t^{\prime}}\right. \\
& \left.-\left[H\left(t^{\prime} ; F_{1}\right)-H\left(t_{i} ; F_{1}\right)\right]\right\} d t^{\prime},
\end{aligned}
$$

and its derivative is

$$
\begin{aligned}
\frac{d S}{d F_{1}}= & -\left[\mathbf{p}\left(t_{i} ; F_{1}\right) \cdot \frac{\partial \mathbf{q}\left(t_{i} ; F_{1}\right)}{\partial t_{i}}\right]\left[\frac{d t_{i}\left(F_{1}\right)}{d F_{1}}\right] \\
& +\int_{t_{i}\left(F_{1}\right)}^{t}\left[\frac{\partial \mathbf{p}\left(t^{\prime} ; F_{1}\right)}{\partial F_{1}} \cdot \frac{\partial \mathbf{q}\left(t^{\prime} ; F_{1}\right)}{\partial t^{\prime}}\right. \\
& +\mathbf{p}\left(t^{\prime} ; F_{1}\right) \cdot \frac{\partial^{2} \mathbf{q}\left(t^{\prime} ; F_{1}\right)}{\partial t^{\prime} \partial F_{1}}-\frac{\partial H\left(t^{\prime} ; F_{1}\right)}{\partial \mathbf{p}} \cdot \frac{\partial \mathbf{p}\left(t^{\prime} ; F_{1}\right)}{\partial F_{1}} \\
& \left.-\frac{\partial H\left(t^{\prime} ; F_{1}\right)}{\partial \mathbf{q}} \cdot \frac{\partial \mathbf{q}\left(t^{\prime} ; F_{1}\right)}{\partial F_{1}}-\frac{\partial H\left(t^{\prime} ; F_{1}\right)}{\partial F_{1}}\right] d t^{\prime} . \quad(\mathrm{C} 2
\end{aligned}
$$

The first term is the boundary term (due to the change in the lower limit of integration) and everything else comes from differentiating under the integral sign. There are other terms, involving $H\left(t_{i} ; F_{1}\right)$, but their derivatives all add to zero because the initial value of $H$ is fixed independent of $F_{1}$.
Inside the integral, the first and third terms cancel. Integrating the second term by parts, we obtain

$$
\begin{array}{rl}
\int_{t_{i}\left(F_{1}\right)}^{t} & \mathbf{p}\left(t^{\prime} ; F_{1}\right) \frac{\partial^{2} \mathbf{q}\left(t^{\prime} ; F_{1}\right)}{\partial t^{\prime} \partial F_{1}} d t^{\prime} \\
= & \left.\mathbf{p}\left(t^{\prime} ; F_{1}\right) \cdot \frac{\partial \mathbf{q}\left(t^{\prime} ; F_{1}\right)}{\partial F_{1}}\right|_{t^{\prime}=t_{i}\left(F_{1}\right)} ^{t^{\prime}=t} \\
& -\int_{t_{i}\left(F_{1}\right)}^{t} \frac{\partial \mathbf{p}\left(t^{\prime} ; F_{1}\right)}{\partial t^{\prime}} \cdot \frac{\partial \mathbf{q}\left(t^{\prime} ; F_{1}\right)}{\partial F_{1}} d t^{\prime} .
\end{array}
$$

The integral in Eq. (C3) cancels the fourth term under the integral in Eq. (C2). The upper-boundary term in Eq. (C3) vanishes because the final point is fixed: $\partial \mathbf{q}\left(t ; F_{1}\right) / \partial F_{1}=0$. The lower-boundary term in Eq. (C3) combines with the boundary term in Eq. (C2),

$$
-\mathbf{p}\left(t_{i} ; F_{1}\right) \cdot\left[\frac{\partial \mathbf{q}\left(t_{i}\left(F_{1}\right) ; F_{1}\right)}{\partial t_{i}} \frac{\partial t_{i}\left(F_{1}\right)}{\partial F_{1}}+\frac{\partial \mathbf{q}\left(t_{i}\left(F_{1}\right) ; F_{1}\right)}{\partial F_{1}}\right]=0
$$

by Eq. (C1). We are left with an expression for the perturbation to the action,

$$
\frac{d S\left(F_{1}\right)}{d F_{1}}=-\int_{t_{i}\left(F_{1}\right)}^{t} \frac{\partial H\left(t^{\prime} ; F_{1}\right)}{\partial F_{1}} d t^{\prime},
$$

from which Eq. (4.1) follows. The derivative of the Hamiltonian is integrated along the unperturbed trajectory.

Let us now evaluate $\Delta S$ for our system. From Eq. (1.1),

$$
\left.\frac{\partial H\left(t^{\prime} ; F_{1}\right)}{\partial F_{1}}\right|_{F_{1}=0}=-e z_{t}\left(t^{\prime}\right) \sin \left(\omega t^{\prime}\right),
$$

where $z_{t}\left(t^{\prime}\right)$ is the unperturbed trajectory that arrives at point $\mathbf{q}_{f}$ at time $t^{\prime}=t$. But when $F_{1}=0$, the shape of the orbit does not depend on the return time, so let us write

$$
z_{0}(\tau) \equiv z_{t}(\tau+t-T), \quad 0 \leqslant \tau \leqslant T,
$$

where $z_{0}(\tau)$ describes the shape of the unperturbed orbit, but its argument always runs from 0 to $T$. Equation (4.2) follows trivially.
[1] (a) M. L. Du and J. B. Delos, Phys. Rev. A 38, 1896 (1988); (b) 38, 1913 (1988).

[2] E. B. Bogomolny, Zh. Éksp. Teor. Fiz. 96, 487 (1989) [Sov. Phys. JETP 69, 275 (1989)]; D. Wintgen and H. Friedrich, Phys. Rev. Lett. 57, 571 (1986); U. Eichmann, K. Richter, D. Wintgen, and W. Sandner, ibid. 61, 2438 (1988); G. Tanner, P. Scherer, E. B. Bogomolny, B. Eckhardt, and D. Wintgen, ibid. 67, 2410 (1991); D. Wintgen and H. Friedrich, Phys. Rev. A 36, 131 (1987); K. Muller, A. Honig, and D. Wintgen, ibid. 47, 3593 (1993).

[3] J. Gao and J. B. Delos, Phys. Rev. A 46, 1455 (1992) [also J.
Gao, J. B. Delos, and M. Baruch, ibid. 46, 1449 (1992)].

[4] J.-M. Mao and J. B. Delos, Phys. Rev. A 45, 1746 (1992); J. Main, G. Wiebusch, K. Welge, J. Shaw, and J. B. Delos, ibid. 49, 847 (1994); J.-M. Mao, K. A. Rapelje, S. J. Blodgett-Ford, J. B. Delos, A. Koenig, and H. Rinneberg, ibid. 48, 2117 (1993); M.-L. Du and J. B. Delos, ibid. 38, 5609 (1988); A. D. Peters and J. B. Delos, ibid. 47, 3020 (1993); 47, 3036 (1993); J. Gao and J. B. Delos, ibid. 49, 869 (1994); A. D. Peters, C. Jaffe, and J. B. Delos, Phys. Rev. Lett. 73, 2825 (1995); D. A. Sadovskii, J. A. Shaw, and J. B. Delos, ibid. 75, 2120 (1994); J. Goetz and J. B. Delos, in Quantum Dynamics of Chaotic 
Systems; Proceedings of the Third Drexel Symposium on Quantum Nonintegrability, Philadelphia, edited by J. Yuan, D. Feng, and G. Zaslavsky (Gordon and Breach, Langhorne, PA, 1993); J.-M. Mao, J. Shaw, and J. B. Delos, J. Stat. Phys. 68, 51 (1992); K. R. Meyer, J. B. Delos, and J.-M. Mao, Fields Inst. Commun. 8, 93 (1996).

[5] P. A. Dando, T. S. Monteiro, D. Delande, and K. T. Taylor, Phys. Rev. Lett. 74, 1099 (1995); F. Penent, D. Delande, and J. C. Gay, Phys. Rev. A 37, 4707 (1988); M. Kus, F. Haake, and D. Delande, Phys. Rev. Lett. 71, 2167 (1993).

[6] G. Raithel, M. Fauth, and H. Walther, Phys. Rev. A 44, 1898 (1991); 47, 419 (1993); G. Raithel, H. Held, L. Marmet, and H. Walther, J. Phys. B 27, 2849 (1994); G. Raithel and M. Fauth, ibid. 28, 1687 (1995); R. C. Hilborn, L. R. Hunter, K. Johnson, S. K. Peck, A. Spencer, and J. Watson, Phys. Rev. A 50, 2467 (1994); E. Flöthmann, Ph.D. thesis, University of Bielefeld, 1994; R. Ubert, Ph.D. thesis, University of Bielefeld, 1995; E. A. Solov'ev, Zh. Éksp. Teor. Fiz. 82, 1762 (1982) [Sov. Phys. JETP 55, 1017 (1982)]; 85, 109 (1983) [58, 63 (1983)]; J. von Milczewski and T. Uzer, Phys. Rev. E 55, 6540 (1997); Related works are J. von Milczewski, G. H. F. Dierckson, and T. Uzer, Phys. Rev. Lett. 76, 2890 (1996); M. J. Gourlay, T. Uzer, and D. Farrelly, Phys. Rev. A 47, 3113 (1993); 48, 2508(E) (1993); J. von Milczewski, G. H. F. Diercksen, and T. Uzer, Int. J. Bifurcation Chaos Appl. Sci. Eng. 4, 905 (1994); D. Sadovskii and B. Zhilinskii, Phys. Rev. A 57, 2867 (1998).

[7] J. A. Yeazell and C. R. Stroud, Jr., Phys. Rev. Lett. 60, 1494 (1988); J. A. Yeazell, M. Mallalieu, and C. R. Stroud, Jr., ibid. 64, 2007 (1990); J. A. Yeazell, G. Raithel, L. Marmet, H. Held, and H. Walther, ibid. 70, 2884 (1993); L. Marmet, H. Held, G. Raithel, J. A. Yeazell, and H. Walther, ibid. 72, 3779 (1994); Xin Chen and J. A. Yeazell, ibid. 81, 5772 (1998); G. M. Lankhuijzen, F. Robicheaux, and L. D. Noordam, ibid. 79, 2427 (1997); R. B. Vrijen, G. M. Lankhuijzen, and L. D. Noordam, ibid. 79, 617 (1997); G. M. Lankhuijzen and L. D. Noordam, Nucl. Instrum. Methods Phys. Res. A 375, 651 (1996); Opt. Commun. 129, 361 (1996).

[8] M. Courtney, H. Jiao, N. Spellmeyer, D. Kleppner, J. Gao, and J. B. Delos, Phys. Rev. Lett. 74, 1538 (1995); also J. A. Shaw, J. B. Delos, M. Courtney, and D. Kleppner, Phys. Rev. A 52, 3695 (1995).
[9] N. Spellmeyer, D. Kleppner, M. R. Haggerty, V. Kondratovich, J. B. Delos, and J. Gao, Phys. Rev. Lett. 79, 1650 (1997).

[10] M. R. Haggerty, N. Spellmeyer, D. Kleppner, and J. B. Delos, Phys. Rev. Lett. 81, 1592 (1998).

[11] M. Protopapas, A. Sanpera, P. L. Knight, and K. Burnett, Phys. Rev. A 52, R2527 (1995); T. Zuo and A. D. Bandrauk, ibid. 54, 3254 (1996); K. J. Schafer and K. C. Kulander, ibid. 45, 8026 (1992); R. M. Potvliege and P. H. G. Smith, ibid. 49, 3110 (1994); E. Charron, A. Giusti-Suzor, and F. H. Mies, ibid. 49, R641 (1994); S. Bivona, R. Burlon, and C. Leone, ibid. 48, R3441 (1993); N.-Y. Du, A. F. Starace, and N. A. Cherepkov, ibid. 48, 2413 (1993).

[12] E. J. Galvez, B. E. Sauer, L. Moorman, P. M. Koch, and D. Richards, Phys. Rev. Lett. 61, 2011 (1988); J. E. Bayfield, G. Casati, I. Guarneri, and D. W. Sokol, ibid. 63, 364 (1989); D. Farrelly and T. Uzer, Phys. Rev. A 38, 5902 (1988); R. V. Jensen, M. M. Sanders, M. Saraceno, and B. Sundaram, Phys. Rev. Lett. 63, 2771 (1989); R. V. Jensen, S. M. Susskind, and M. M. Sanders, ibid. 62, 1476 (1989); J. M. Hettema, P. Fu, and T. F. Gallagher, Phys. Rev. A 41, 6555 (1990); R. Blumel, ibid. 49, 4787 (1994); O. Benson, A. Buchleitner, G. Raithel, M. Arndt, R. N. Mantegna, and H. Walther, ibid. 51, 4862 (1995); M. Wojcik, J. Zakrzewski, and K. Rzazewski, ibid. 52, R2523 (1995).

[13] This work could straightforwardly be generalized to $m \neq 0$ by allowing the initial wave function to be $\phi$ dependent. The classical trajectories would be approximately unchanged, though their weights and phases would be modified. See, for example, J. Goetz and J. B. Delos (listed in Ref. [4]); G. J. Kuik, A. Kips, W. Vassen, and W. Hogervorst, J. Phys. B 29, 2159 (1996); A. Kips, W. Vassen, W. Hogervorst, and P. A. Dando, Phys. Rev. A 58, 3043 (1998); A. Kips, Ph.D. thesis, Vrije Universiteit Amsterdam, 1999.

[14] V. P. Maslov and M. V. Fedoriuk, Semiclassical Approximation in Quantum Mechanics (Reidel, Boston, 1981).

[15] M. C. Gutzwiller, Chaos in Classical and Quantum Mechanics (Springer-Verlag, Berlin, 1990), Chap 2.

[16] N. Spellmeyer and D. Kleppner (private communication).

[17] C. Neumann, R. Ubert, S. Freund, E. Flöthmann, B. Sheehy, K. H. Welge, M. R. Haggerty, and J. B. Delos, Phys. Rev. Lett. 78, 4705 (1997). 